\title{
The mass function of the Arches cluster from Gemini adaptive optics data ${ }^{\star}$
}

\author{
A. Stolte ${ }^{1,2}$, E. K. Grebel ${ }^{1}$, W. Brandner ${ }^{2}$, and D. F. Figer ${ }^{3}$ \\ 1 Max-Planck-Institut für Astronomie, Königstuhl 17, 69117 Heidelberg, Germany \\ e-mail: stolte@mpia-hd.mpg.de; grebel@mpia-hd.mpg.de \\ 2 European Southern Observatory, Karl-Schwarzschild-Str. 2, 85748 Garching, Germany \\ e-mail: wbrandne@eso.org \\ 3 Space Telescope Science Institute, 3700 San Martin Drive, Baltimore, MD 21218, USA \\ e-mail: figer@stsci.edu
}

Received 8 March 2002 / Accepted 30 July 2002

\begin{abstract}
We have analysed high resolution adaptive optics (AO) science demonstration data of the young, massive stellar cluster Arches near the Galactic Center, obtained with the Gemini North telescope in combination with the University of Hawai'i AO system Hokupa'a. The AO $H$ and $K^{\prime}$ photometry is calibrated using HST/NICMOS observations in the equivalent filters $F 160 W$ and $F 205 W$ obtained by Figer et al. (1999). The calibration procedure allows a detailed comparison of the ground-based adaptive optics observations against diffraction limited space-based photometry. The spatial resolution as well as the overall signal-to-noise ratio of the Gemini/Hokupa'a data is comparable to the HST/NICMOS data. The low Strehl ratio of only a few percent is the dominant limiting factor in the Gemini AO science demonstration data as opposed to space-based observations. After a thorough technical comparison, the Gemini and HST data are used in combination to study the spatial distribution of stellar masses in the Arches cluster. Arches is one of the densest young clusters known in the Milky Way, with a central density of $\sim 3 \times 10^{5} M_{\odot} \mathrm{pc}^{-3}$ and a total mass of about $10^{4} M_{\odot}$. A strong colour gradient is observed over the cluster field. The visual extinction increases by $\Delta A_{\mathrm{V}} \sim 10$ mag over a distance of $15^{\prime \prime}$ from the cluster core. Extinction maps reveal a low-extinction cavity in the densest parts of Arches $\left(R \leq 5^{\prime \prime}\right)$, indicating the depletion of dust due to stellar winds or photo-evaporation. We correct for the change in extinction over the field and show that the slope of the mass function is strongly influenced by the effects of differential extinction. We obtain present-day mass function slopes of $\Gamma \sim-0.8 \pm 0.2$ in the mass range $6<M<65 M_{\odot}$ from both data sets. The spatial analysis reveals a steepening of the mass function slope from close to zero in the cluster center to $\Gamma \sim-1.7 \pm 0.7$ at $R>10^{\prime \prime}$, in accordance with a Salpeter slope $(\Gamma=-1.35)$. The bias in the mass function towards high-mass stars in the Arches center is a strong indication for mass segregation. The dynamical and relaxation timescales for Arches are estimated, and possible mass segregation effects are discussed with respect to cluster formation models.
\end{abstract}

Key words. open clusters and associations: individual: Arches - stars: luminosity function, mass function - stars: early-type stars: formation - ISM: dust, extinction - instrumentation: adaptive optics

\section{Introduction}

The Galactic Center (GC) is the most extreme star forming environment within the Milky Way. High stellar and gas densities, turbulent motion, tidal torques exerted by the steep gravitational potential, magnetic fields and an intense radiation field determine the physical environment of star formation in the GC region. Although disruptive forces exerted by the gravitational and radiation fields counteract the agglomeration of material, the high gas and dust densities cause star formation in the GC

Send offprint requests to: A. Stolte, e-mail: stolte@mpia-hd.mpg.de

* Based on observations obtained with the Gemini North Telescope and the NASA/ESA Hubble Space Telescope. environment to be most efficient. In particular, the formation of high mass stars and massive clusters is more successful than in any other region of the Milky Way.

A detailed study of star formation processes and the stellar content of the GC region has until recently been limited to the brightest and most massive stars due to the large amount of extinction $\left(A_{\mathrm{V}} \sim 30 \mathrm{mag}\right)$ along the line of sight. Additional constraints are imposed due to the spatial resolution at the GC distance of $\sim 8 \mathrm{kpc}(D M=14.47 \pm 0.08 \mathrm{mag}$, e.g., McNamara et al. 2000), much farther than nearby star forming regions such as the Orion or $\rho$ Ophiuchi star forming complexes, which have been studied in greater detail to date. Only with the advent of deep, high resolution near-infrared instruments, the analysis of 
stellar populations in young star clusters near the GC has become feasible.

During the past few years, it has become evident that three out of four young starburst clusters known in the Milky Way are located in the GC region - namely, the Arches and Quintuplet clusters, as well as the Galactic Center Cluster itself. With a cluster age of only a few Myr for Arches and Quintuplet, the question arises how many clusters do actually form in the densest environment of the Milky Way. The 2MASS database yielded new insights into the estimated number of star clusters hidden in the dense stellar background. Dutra \& Bica (2000, 2001) report the detection of new cluster candidates of various ages located in the innermost $200 \mathrm{pc}$ of the Galaxy found in 2MASS. Numerical simulations by Portegies Zwart et al. (2001) suggest that clusters with properties similar to the massive Arches and Quintuplet may have formed in the past in the innermost $200 \mathrm{pc}$, but were then dispersed and are now indistinguishable from the dense stellar background. As dynamical evolution timescales are short due to the strong tidal field in the GC region (Kim et al. 1999), young star clusters are disrupted quickly after formation, contributing to the Galactic bulge population. Thus, only the youngest clusters remain intact for the study of star formation in this extraordinary environment.

The Arches cluster, at a projected distance of only $25 \mathrm{pc}$ from the GC (assuming a heliocentric distance of $8 \mathrm{kpc}$ to the GC), is one of the most massive young clusters known in the Milky Way. With an estimated mass of about $10^{4} M_{\odot}$ and a central density of $3 \times 10^{5} M_{\odot} \mathrm{pc}^{-3}$, Arches is the densest young star cluster (YC) known (Figer et al. 1999). From physical properties of Wolf-Rayet stars, the age of the cluster is estimated to be between 2 and 4.5 Myr (Blum et al. 2001). The stellar content of Arches has been studied by Figer et al. (1999) using HST/NICMOS data. They derived a shallow initial mass function in the range $6<M<120 M_{\odot}$ with a slope of $\Gamma=-0.7 \pm 0.1$, but with significant flattening observed in the innermost part of the cluster $(\Gamma=-0.1 \pm 0.2)$.

Most young star clusters and associations in the Milky Way display a mass function close to a Salpeter (1955) power law with a slope of $\Gamma=-1.35$. Several such star forming regions have been studied by Massey et al. (1995a), yielding slopes in the range $-0.7<\Gamma<-1.7$ with an average of $-1.1 \pm 0.1$, which leads these authors to conclude that within the statistical limits no deviation from a Salpeter slope is observed.

A flat mass function as observed in Arches implies an overpopulation of the high-mass end as compared to "normal" clusters. The special physical conditions in the GC region have been suggested to enhance the formation of massive stars, thereby resulting in a flattened mass function (Morris 1993). The formation of high-mass stars in itself poses serious problems for the standard core collapse and subsequent accretion model, as radiation pressure from the growing star is capable of reversing the gas infall as soon as the mass is in excess of $10 M_{\odot}$ (Yorke \& Krügel 1977). Assuming disk accretion instead of spherical infall, the limiting mass may be increased to $15 M_{\odot}$ (Behrend \& Maeder 2001), still far below the mass observed in O-type stars. Various scenarios are suggested to circumvent this problem. Simulations with enhanced accretion rates and collision probabilities in dense cluster centers (Bonnell et al. 1998), as well as growing accretion rates depending on the mass of the accreting protostar (Behrend \& Maeder 2001), allow stars of up to $100 M_{\odot}$ to form in the densest regions of a rich star cluster. In case of the GC environment, a higher gas density may lead to a higher accretion rate and/or to a longer accretion process in the protostellar phase. As long as the gravitational potential is strongly influenced by the amount of gas associated with the cluster, gas infall causes a decrease in cluster radius and subsequent increase in the collision rate, reinforcing the formation of high-mass stars. Physical processes such as gravitational collapse or cloud collisions scale with the square root of the local density, $\sqrt{\rho}$ (Elmegreen 1999, 2001), causing an enhanced star formation rate (SFR) in high density environments. Elmegreen (2001) shows that the total mass as well as the maximum stellar mass in a cluster strongly depends on the SFR and local density. This is confirmed by observations of high-mass stars found predominantly in the largest star forming clouds (Larson 1982).

Both the growing accretion and the collision scenario predict the high-mass stars to form in the densest central region of a cluster, leading to primordial mass segregation, which may be evidenced in a flat mass function in the dense cluster center. As an additional physical constraint, both scenarios require the lower-mass stars to form first, and the highest-mass stars last in the cluster evolution process. As the strong UV-radiation field originating from hydrogen ignition in high-mass stars expells the remaining gas from the cluster center, the accretion process should be halted immediately after high-mass star formation.

The short dynamical timescales of compact clusters are, however, influencing the spatial distribution of stellar masses as well. On the one hand, high-mass stars are dragged into the cluster center due to the gravitational potential of the young cluster. On the other hand, low-mass stars may easily be flung out of the cluster due to interaction processes, especially given star densities as high as in the Arches cluster. The result of these processes would also be a flat mass function in the cluster center, steepening as one progresses outwards due to dynamical mass segregation. Dynamical segregation is predicted to occur within one relaxation time (Bonnell \& Davies 1998), which for compact clusters is only one to a few Myr, and should thus be well observable in Arches in the form of a spatially varying mass function.

In addition to the internal segregation process, the external GC tidal field exerts shear forces tearing apart the cluster entity. $N$-body simulations by Kim et al. (2000) yield tidal disruption timescales as short as 10 to $20 \mathrm{Myr}$ in the GC tidal field. We expect to find a mixture of all these effects in the Arches cluster.

We have analysed adaptive optics (AO) data obtained under excellent seeing conditions with the Gemini North $8 \mathrm{~m}$ telescope in combination with the University of Hawai'i (UH) AO system Hokupa'a. We are investigating the presence of radial variations in the mass distribution within the Arches cluster. We compare our ground-based results in detail to the HST/NICMOS data presented in Figer et al. (1999, hereafter FKM), discussing possible achievements and limitations of ground-based, high-resolution adaptive optics versus spacebased deep NIR photometry. 
In Sect. 2, we will introduce the data and describe the reduction and calibration processes. In this context, a thorough investigation of the quality of ground-based adaptive optics photometry as compared to space-based diffraction limited observations will be presented. The photometric results derived from colour-magnitude diagrams and extinction maps will be discussed in Sect. 3. A comparison of Gemini and HST luminosity functions will be given in Sect. 4 . The mass functions will be derived in Sect. 5, and their spatial variation will be discussed with respect to cluster formation scenarios. We will estimate the relevant timescales for cluster evolution for the Arches cluster in Sect. 6, and discuss the implication on the dynamical evolution of Arches. We will summarise our results in Sect. 7.

\section{Observations and data reduction}

\subsection{Gemini/Hokupa'a data}

We analysed $H$ and $K^{\prime}$ images of the Arches cluster center obtained in the course of the Gemini science demonstration at the Gemini North 8 m telescope located on Mauna Kea, Hawai'i, at an altitude of $4200 \mathrm{~m}$ above sea level.

Gemini is an alt-azimuth-mounted telescope with a monolithic primary mirror and small secondary mirror optimised for IR observations. The telescope is always used in Cassegrain configuration with instruments occupying either the upward looking Cassegrain port or one of three sideward facing ports. The University of Hawai'i adaptive optics (AO) system Hokupa'a is a 35 element curvature sensing AO system (Graves et al. 2000), which typically delivers Strehl ratios between 5\% and $25 \%$ in the $K$-band.

Hokupa'a is operated with the near-infrared camera QUIRC (Hodapp et al. 1996), equipped with a $1024 \times$ 1024 pixel $\mathrm{HgCdTe}$ array. The plate scale is 19.98 milliarcsec per pixel, yielding a field of view (FOV) of 20.2". The images are shown in Fig. 1, along with the HST/NICMOS images used for calibration.

The observations were carried out between July 3 and 30, 2000. 12 individual $60 \mathrm{~s}$ exposures, dithered in a 4 position pattern with an offset of 16 pixels $\left(0.32^{\prime \prime}\right)$ between subsequent frames, were coadded to an $H$-band image with a total integration time of $720 \mathrm{~s}$. In $K^{\prime}$, the set of 34 dithered $30 \mathrm{~s}$ exposures obtained under the best observing conditions was coadded to yield a total exposure time of $1020 \mathrm{~s}$. The full width at half maximum $(F W H M)$ of the point spread function (PSF) was 9.5 pixels $\left(0.19^{\prime \prime}\right)$ in $H$ and 6.8 pixels $\left(0.135^{\prime \prime}\right)$ in $K^{\prime}$. The observations were carried out at an airmass of 1.5 , the lowest airmass at which the Galactic Center can be observed from Hawai'i.

The $H$-band data were oversampled, and $2 \times 2$ binning was applied to improve the effective signal-to-noise ratio per resolution element, allowing a more precise PSF fit. A combination of long and short exposures has been used to increase the dynamic range. For the short exposures, 3 frames with $1 \mathrm{~s}$ exposure time have been coadded in $H$, and 16 such frames in $K^{\prime}$. See Table 1 for the observational details. In the long exposures, the limiting magnitudes were about $21 \mathrm{mag}$ in $H$ and 20 mag in $K^{\prime}$. Note that the completeness limit in the crowded regions was significantly lower. The procedure used for completeness correction will be described in detail in Sect. 2.1.6.

\subsubsection{Data reduction}

The data reduction was carried out by the Gemini data reduction team, F. Rigaut, T. Davidge, R. Blum, and A. Cotera. The procedure as outlined in the science demonstration report ${ }^{1}$ was as follows: sky images, obtained after the short observation period when the Galactic Center was in culmination, were averaged using median clipping for star rejection, and then subtracted from the individual images. The frames were then flatfielded and corrected for bad pixels and cosmic ray hits. After inspecting the individual frames with respect to signal-to-noise ratio and resolution, and background adjustment, the images with sufficient quality were combined using sigma clipped averaging. The final images were scaled to counts per second. For the analysis presented in this paper, this set of images reduced by the Gemini reduction team has been used.

\subsubsection{Photometry}

The photometry was performed using the IRAF $^{2}$ (Tody 1993) DAOPHOT implementation (Stetson 1987). Due to the wavelength dependence of the adaptive optics correction and anisoplanatism ${ }^{3}$ over the field, the $H$ and $K^{\prime}$ data have been treated differently for PSF fitting. While in $H$ the PSF radius increases significantly with distance from the guide star, with a radially varying FWHM in the range of $0.18^{\prime \prime}$ to $0.23^{\prime \prime}$ (see the science demonstration data description), the $K^{\prime}$ PSF was nearly constant over the field $\left(0.125^{\prime \prime}\right.$ to $\left.0.135^{\prime \prime}\right)$. This behaviour is expected from an $\mathrm{AO}$ system, as the isoplanatic angle $\theta_{0} \theta_{0}$ varies as $\lambda^{6 / 5}$, yielding a 1.4 times larger $\theta_{0}$ in $K^{\prime}$ than in $H$, resulting in a more uniform PSF in $K^{\prime}$ across the field of view.

As obscuration due to extinction decreases with increasing wavelength, many more stars are detected in $K^{\prime}$ than in $H$. For comparison, the number of objects found with $K^{\prime}<20 \mathrm{mag}$ and uncertainty $\sigma_{K^{\prime}}<0.2$ mag was 1017 (1020 s effective exposure time), while for $H<20 \mathrm{mag}$ and $\sigma_{\mathrm{H}}<0.2 \mathrm{mag}$ we detected only 391 objects ( $720 \mathrm{~s}$ effective exposure time), where in both cases visual inspection led to the conclusion that objects with photometric uncertainties below 0.2 mag were real detections. On the other hand, the increased stellar number density

\footnotetext{
1 The description of the Galactic Center dataset can be found at http://www.gemini .edu/gallery/observing/release_doc/ manual.html, and the Gemini North science demonstration data are publicly available at

http://www.gemini .edu/sciops/data/dataSV.html

2 IRAF is distributed by the National Optical Astronomy Observatories, which are operated by the Association of Universities for Research in Astronomy, Inc., under cooperative agreement with the National Science Foundation.

3 The anisoplanatism describes the degradation of the AO correction due to differences between wave fronts coming from different directions.

4 The isoplanatic angle is defined as the angular distance for which the rms error of the wave front is less than 1 radian.
} 


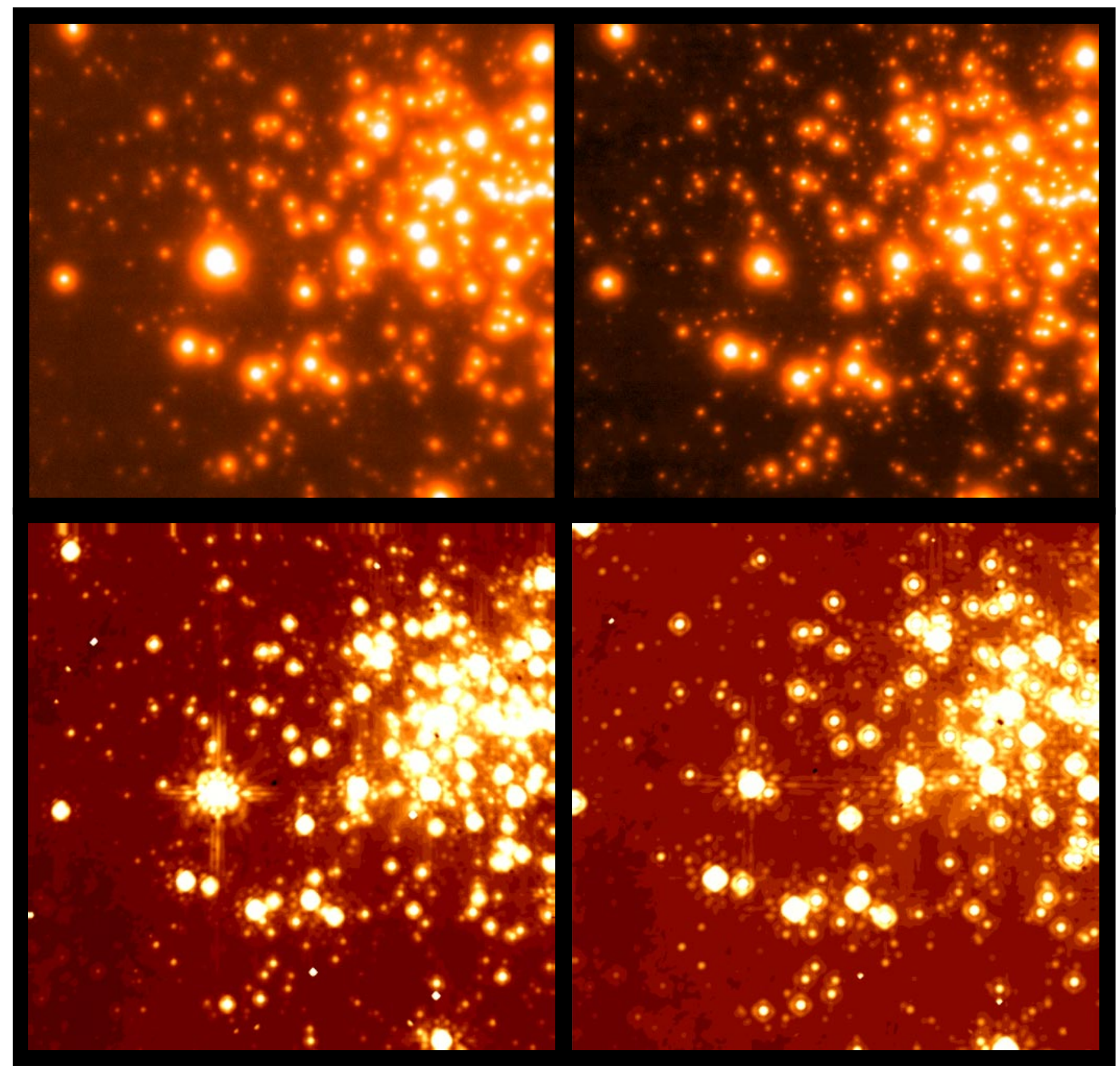

Fig. 1. Upper panels: Gemini/Hokupa'a $H$ (left) and $K^{\prime}$ (right) images, $20^{\prime \prime} \times 20^{\prime \prime}(0.8 \mathrm{pc} \times 0.8 \mathrm{pc})$ with a spatial resolution of less than $0.2^{\prime \prime}$. North is up and East is to the right. Lower panels: HST/NICMOS $F 160 W$ and $F 205 W$ images from Figer et al. (1999), geometrically transformed to the Gemini FOV.

in $K^{\prime}$ leads to increased crowding effects, such that we decided to use a non-variable PSF for the $K^{\prime}$-band data after thorough investigation of the results of a quadratically, linearly or nonvarying PSF. It turns out that, due to a lack of isolated stars for the determination of the PSF variation across the field, the mean uncertainty is lower and the number of outliers with unacceptably large uncertainties reduced when a non-variable PSF is used. Thus, the 5 most isolated stars on the $K^{\prime}$ image, which were well spread out over the field, were used to derive the median averaged PSF of the long exposure. In the case of the short exposure, where due to the very short integration time faint stars are indistinguishable from the background, leaving more "uncrowded" stars to derive the shape of the PSF, 7 isolated stars could be used. In $K^{\prime}$, the best fitting function was an elliptical Moffat-function with $\beta=2.5$.

Due to the lower detection rate on the $H$-band image crowding is less severe, while the PSF exhibits more pronounced spatial variations than in $K^{\prime}$. We thus used the quadratically variable option of the DAOPHOT psf and allstar tasks for our $H$-band images, with 27 stars to determine a median averaged PSF function and residuals. The best fitting function was a Lorentz function on the binned $H$-band image. In both filters, the average $F W H M$ of the data has been used as the PSF fitting 
Table 1. Gemini/Hokupa'a and HST/NICMOS observations.

\begin{tabular}{llrrrrrrr}
\hline \hline Date & Filter & single exp. & $n_{\text {exp }}$ & exp. total & det. limit & $\sigma_{\text {back }}$ & resolution & diffraction limit \\
\hline $05 / 07 / 2000$ & $H$ & $1 \mathrm{~s}$ & 3 & $3 \mathrm{~s}$ & $18.5 \mathrm{mag}$ & 7.74 & $0.17^{\prime \prime}$ & $0.05^{\prime \prime}$ \\
$05 / 07 / 2000$ & $H$ & $60 \mathrm{~s}$ & 12 & $720 \mathrm{~s}$ & $21 \mathrm{mag}$ & 0.19 & $0.20^{\prime \prime}$ & $0.05^{\prime \prime}$ \\
$30 / 07 / 2000$ & $K^{\prime}$ & $1 \mathrm{~s}$ & 16 & $16 \mathrm{~s}$ & $17.5 \mathrm{mag}$ & 3.73 & $0.12^{\prime \prime}$ & $0.07^{\prime \prime}$ \\
$09 / 07 / 2000$ & $K^{\prime}$ & $30 \mathrm{~s}$ & 34 & $1020 \mathrm{~s}$ & $20 \mathrm{mag}$ & 0.22 & $0.13^{\prime \prime}$ & $0.07^{\prime \prime}$ \\
\hline & \multicolumn{7}{c}{ HST } \\
\hline $14 / 09 / 1997$ & $F 160 W$ & & $256 \mathrm{~s}$ & $21 \mathrm{mag}$ & 0.04 & $0.18^{\prime \prime}$ & $0.17^{\prime \prime}$ \\
$14 / 09 / 1997$ & $F 205 W$ & $256 \mathrm{~s}$ & $20 \mathrm{mag}$ & 0.15 & $0.22^{\prime \prime}$ & $0.21^{\prime \prime}$ \\
\hline
\end{tabular}

radius, i.e., the kernel of the best-fitting PSF function, to derive PSF magnitudes of the stars.

The short exposures have been used to obtain photometry of the brightest stars, which are saturated in the long exposures. The photometry of the long and short exposures agreed well after atmospheric extinction correction in the form of a constant offset had been applied. The saturation limit was 13.0 mag in $H$ and $13.3 \mathrm{mag}$ in $K^{\prime}$. At fainter magnitudes, the photometry of both images was indiscernible within the uncertainties for the bright stars, and the better quality long exposure values were used. Furthermore, a comparison of the bright star photometries was used to estimate photometric uncertainties (see Sect. 2.1.6 and Table 2).

\subsubsection{Photometric calibration}

To transform instrumental into apparent magnitudes, we used the HST/NICMOS photometry of Figer et al. (1999) as local standards. The advantage of this procedure lies in the possibility to correct for remaining PSF deviations over the field, e.g., due to a change in the Strehl ratio with distance from the guide star or due to the increased background from bright star halos in the cluster center. Indeed, as will be discussed below, the spatial distribution of photometric residuals shows a mixture of these effects.

We were able to use approximately 380 stars to derive colour equations. The residuals obtained for these stars after calibration allow a detailed analysis and correction of field variations. The colour equations to transform Gemini instrumental $H$ and $K^{\prime}$ magnitudes to magnitudes in the HST/NICMOS filter system were determined using the IRAF PHOTCAL package, yielding:

$m 160=H_{\text {inst }}+0.001( \pm 0.017) \cdot\left(H-K^{\prime}\right)_{\text {inst }}$

$$
-0.028( \pm 0.031) \mathrm{mag}
$$

$m 205=K_{\mathrm{inst}}^{\prime}+0.023( \pm 0.008) \cdot\left(H-K^{\prime}\right)_{\mathrm{inst}}$

$$
-1.481( \pm 0.016) \mathrm{mag}
$$

where $H_{\text {inst }}$ and $K_{\text {inst }}^{\prime}$ are the Gemini instrumental magnitudes, and $m 160$ and $m 205$ correspond to magnitudes obtained with the NICMOS broadband filters $F 160 W$ and $F 205 W$, respectively. After the transformation had been applied, it turned out that the residual magnitudes in the two independent fitting parameters $H-K^{\prime}$ and $K^{\prime}$ still varied systematically over the field. As can be seen in Fig. 3, the variation is not a simple radial variation increasing with distance from the guide star (GS), but a mixture of displacement from the GS position (shown in the contour map in Fig. 3 as a cross), and the position with respect to the cluster center or bright stars in the field. Fortunately, the variation was well behaved in the $Y$-direction, and could be fitted by a fourth order polynomial. Close inspection showed that two areas on the QUIRC array showed a remaining photometric offset compared to the HST photometry. In the region $400<X<700$ pixels and $Y<250$ pixels, the magnitude was underestimated by $0.1 \mathrm{mag}$. For $X>850$ pixels and $Y>850$ pixels, i.e., the upper right corner, the $K^{\prime}$ magnitude was overestimated by $0.25 \mathrm{mag}$ (however, note that there are only 7 stars in this region). For a discussion of these effects, see Sect. 2.1.4. We corrected for these offsets before deriving the $Y$ correction, which then showed remarkable homogeneity over the entire field. This smooth correction function is probably due to discrepancies in the dome flat field illumination versus sky exposures. The correction was then applied to transformed $K^{\prime}$ and $H-K^{\prime}$ magnitudes, denoted $K_{\text {trans }}^{\prime}$ and $\left(H-K^{\prime}\right)_{\text {trans }}$ from now on. The $H_{\text {trans }}$ magnitude was calculated from the corrected $K_{\text {trans }}^{\prime}$ and $\left(H-K^{\prime}\right)_{\text {trans }}$ values.

An additional advantage of this procedure is the independence on uncertainties in colour transformations at large reddening and non-main sequence colours, as opposed to colour transformations derived from typical main sequence standard stars. Using the HST photometry as local standards, we are naturally in equal colour and temperature regimes, allowing the direct comparison of the Gemini and HST photometry. For most parts of the paper, we remain in the HST/NICMOS system. We use the colour equations obtained in Brandner et al. (2001, hereafter BGB) to transform typical main sequence colours and theoretical isochrone magnitudes into the HST/NICMOS system where indicated. This allows us to transform mainly unreddened main-sequence stars, for which the BGB colour transformations have been established. The only exceptions are the two-colour diagram (Sect. 3.4) and the derivation of the extinction variation from colour gradients (Sect. 3.1), where the extinction law is needed to determine the reddening path. We will use the notation " $m 160$ " and " $m 205$ " as in FKM for magnitudes in the HST/NICMOS filters, and " $H_{\text {trans }}$ " and " $K$ trans" for the Gemini/Hokupa'a data calibrated to the NICMOS 

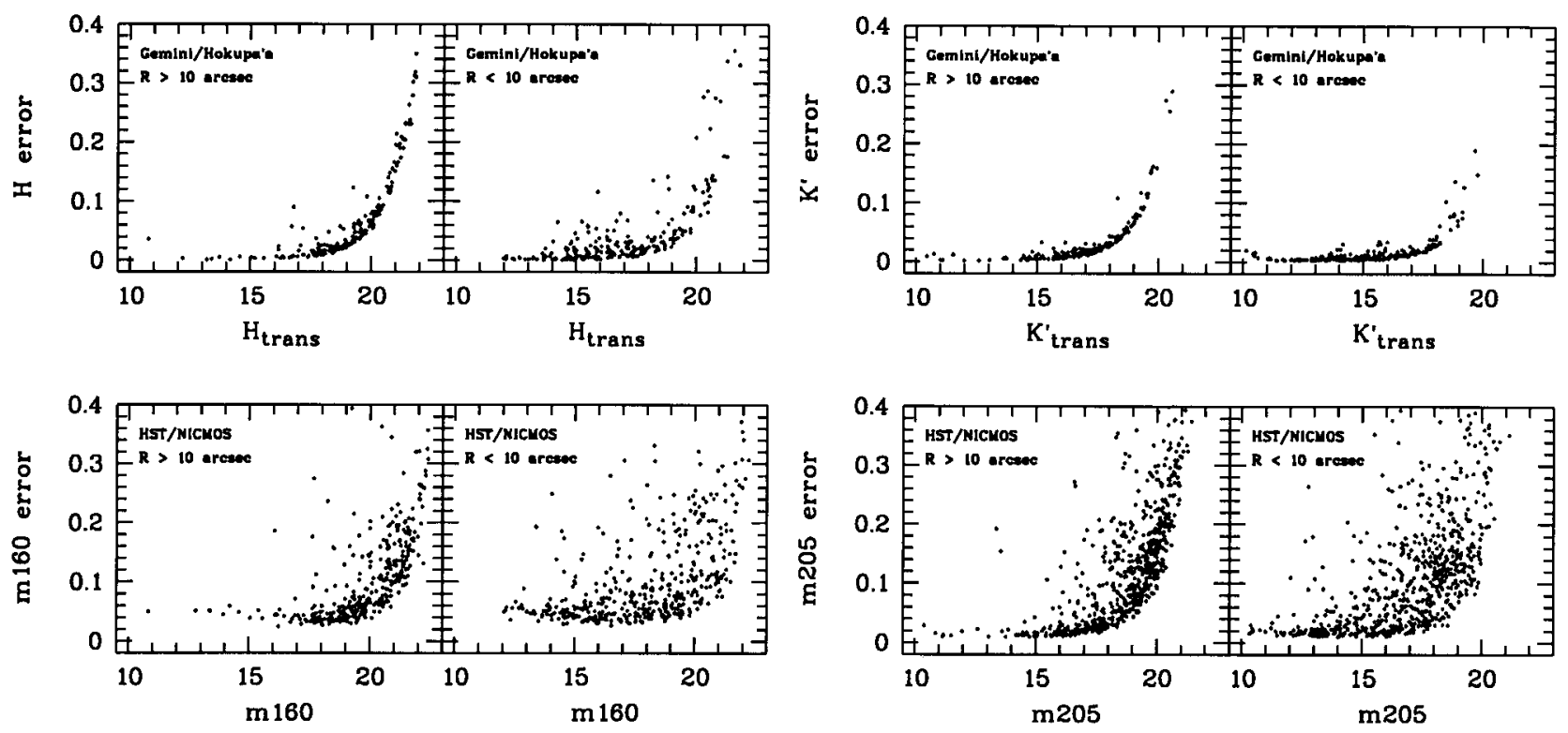

Fig. 2. Formal DAOPHOT photometric uncertainties. Top panel: Gemini/Hokupa'a $H$ and $K^{\prime}$ photometry transformed to HST/NICMOS $F 160 W$ and $F 205 W$ filter magnitudes, $m 160$ and $m 205$. Bottom panel: HST/NICMOS $m 160, m 205$ photometry of the same $20 \times 20^{\prime \prime}$ field (shown in Fig. 1).

photometric system. HST magnitudes transformed to the ground-based 2MASS system will be denoted by $\left(J H K_{\mathrm{s}}\right)_{2 \mathrm{MASS}}$ or simply $J H K_{\mathrm{s}}$.

\subsubsection{Discussion of the residual maps}

The behaviour of the residual of the HST/NICMOS vs. Gemini magnitudes, $m_{\text {NICMOS }}-m_{\text {Gemini,trans }}$, can be analysed in more detail when studying the residual map and the smoothed contour plot. In Fig. 3, positive (negative) residuals correspond to overestimated (underestimated) flux. From the map we denote a general tendency to overestimate the flux. The contour maps show that positive residuals are correlated with the position of bright stars on the $K^{\prime}$ image, both in the crowded cluster center as well as in the area to the lower left, where a band of bright stars is located (see Fig. 1). This is the area where the $K^{\prime}$ magnitudes were found to be underestimated in Sect. 2.1.3, and thus the flux overestimated. This suggests that the increased background due to the uncompensated seeing halos of bright stars (cf. Sect. 2.1.5), inherent to AO observations, causes an overestimation of the flux of bright $\left(K^{\prime} \lesssim 13 \mathrm{mag}\right)$ sources. On the other hand, points with negative residuals are mainly correlated with fainter stars ( $K \gtrsim 16 \mathrm{mag}$ ), suggesting that this enhanced background leads to an oversubtraction of the individually calculated background of nearby fainter stars. The result is an underestimate of the flux of companion stars in the vicinity of bright stars. The correlation of positive residuals with the position of bright stars seems to be less pronounced in the $H$-band image (Fig. 3). In $H$, the distance from the guide star is supposed to be more important due to the smaller size of the isoplanatic angle at shorter wavelengths and consequently more pronounced anisoplanatism. Indeed, the smoothed residual contour plot shows a symmetry in the residuals around the guide star, with close-to-zero residuals in the immediate vicinity of the guide star, where the best adaptive optics correction can be achieved. With increasing distance from the guide star, the residuals increase not only towards the bright cluster, but also to the west (left in Fig. 1) of the field, indicating that remaining distortion effects from the $\mathrm{AO}$ correction are mixed with the problem of the proximity to bright stars as seen in $K^{\prime}$.

\subsubsection{Strehl ratio}

The Strehl ratio $(S R)$ is defined to be the ratio of the observed peak-to-total flux ratio to the peak-to-total flux ratio of a perfect diffraction limited optical system. This definition allows to compare the quality and photometric resolution of different optical systems using a single characteristic quantity.

$S R=\left(F_{\text {peak }} / F_{\text {total }}\right)_{\text {obs }} /\left(F_{\text {peak }} / F_{\text {total }}\right)_{\text {theo }}$

where $F_{\text {peak }}$ is the maximum flux value of the PSF, and $F_{\text {total }}$ is the total flux, including the uncompensated halos induced by the natural seeing. The labels obs and theo refer to the observed and the theoretically expected flux, respectively. Diffraction limited theoretical PSFs have been calculated using the imgen task of the ESO data analysis package eclipse (Devillard 1997). The Strehl ratio in the Gemini $H$-band 720 s exposure is found to be $2.5 \%$ compared to $95 \%$ in the HST $F 160 \mathrm{~W}$ image, and $7 \%$ in $K^{\prime}(1020$ s) compared to $\sim 90 \%$ in $F 205 W$. The low SRs measured in the Gemini science demonstration data indicate that more than $90 \%$ of the light of a star is distributed into the resolution pattern of the AO PSF and the halo around each star induced by the natural seeing. It has turned out that these halos cause a significant limitation to the resolution and depth of the observations in a crowded field, as faint stars can be lost to the enhanced background in the vicinity of bright objects. When comparing the HST and Gemini luminosity functions (Sect. 4), this effect causes the main difference between both data sets. 

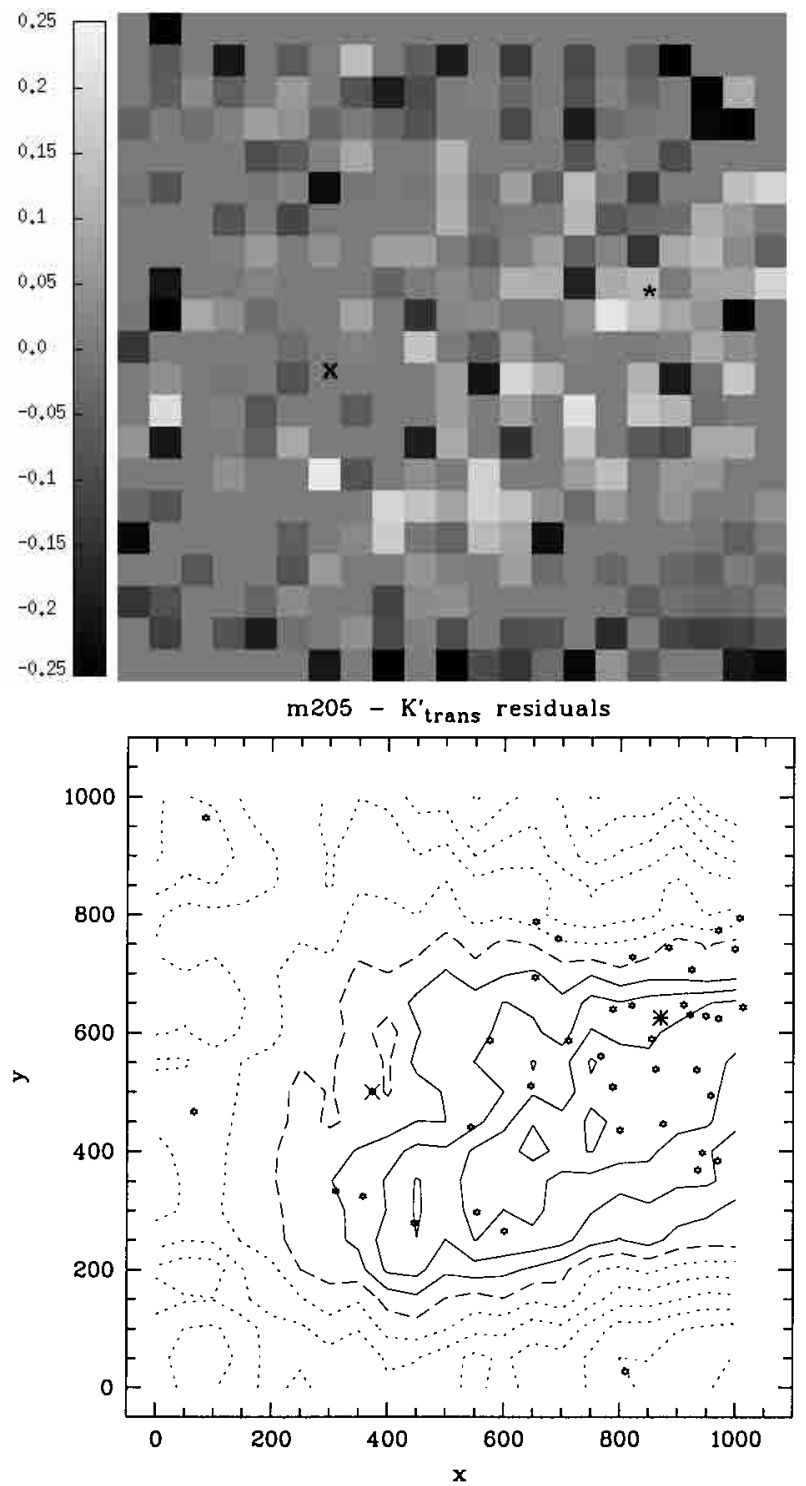
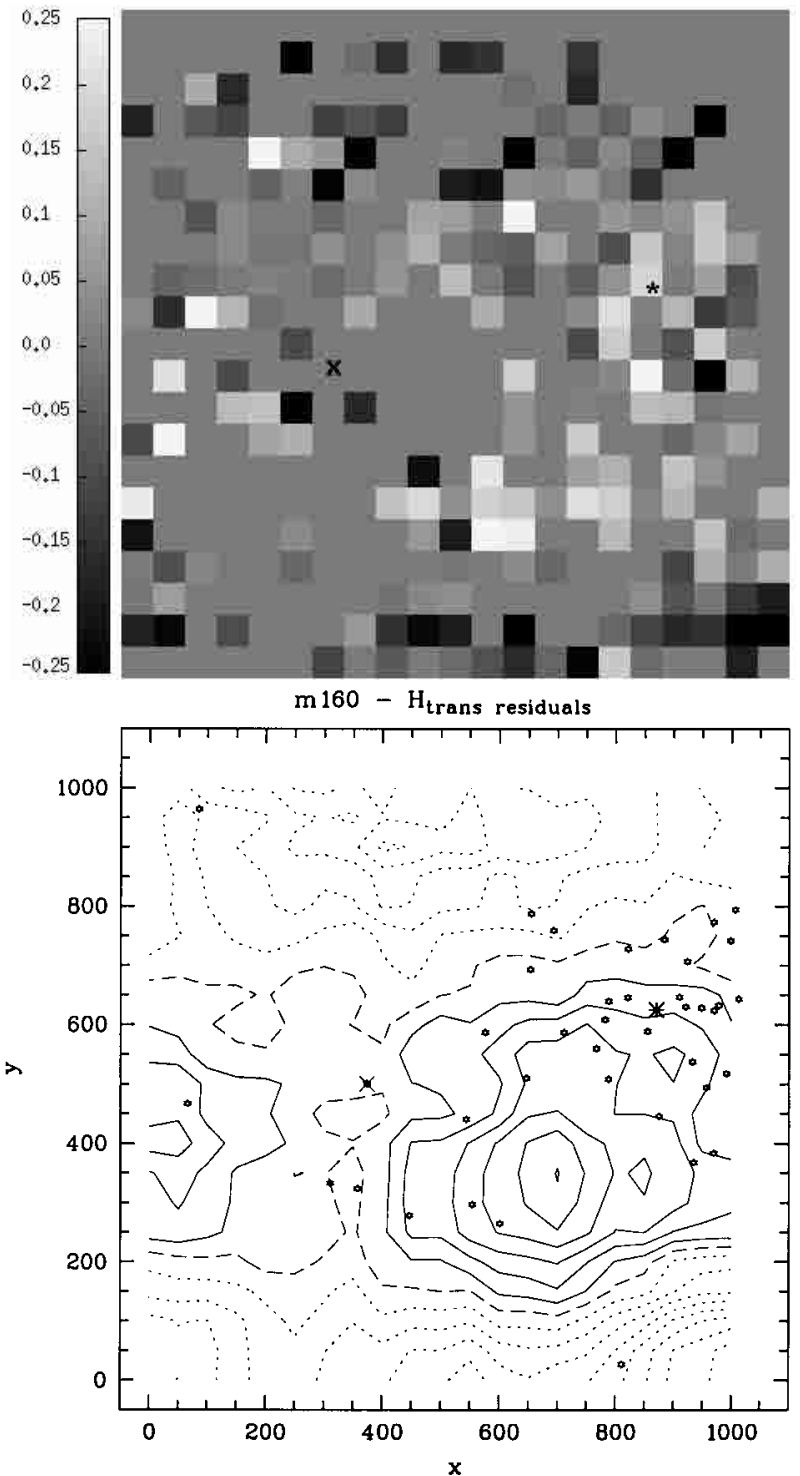

Fig. 3. Map of residuals of NICMOS vs. Gemini photometry (orientation as in Fig. 1). Left panels: $m 205-K_{\text {trans }}^{\prime}$, right panels: $m 160-H_{\text {trans }}$. Stars have been binned into areas of $50 \times 50$ pixels, and the value displayed shows the average of all stars in each bin. Statistical fluctuations are large due to the varying number of stars in each pixel intervall, but the overall trends are clearly visible. The position of the guide star is marked by a cross, the cluster center as determined from the HST/NICMOS F205W image as an asterisk, and stars denote stars resolved in the Gemini images with magnitudes brighter than $K_{\text {trans }}^{\prime}=13 \mathrm{mag}$. The strong correlation between the position of bright stars and positive residuals reveals the tendency of PSF fitting photometry applied to AO data to overestimate the flux of bright sources, and underestimate the flux of faint sources in their vicinity (see text for discussion).

In the case of very low Strehl ratios, the SR does not directly indicate the fraction of the flux concentrated in the FWHM area of the PSF. A much larger fraction of the source flux can be used for PSF fitting in this case, although the spatial resolution is limited by the large FWHM as compared to diffraction limited observations (cf. Table 1). The ratio of the flux in the FWHM kernel of the compensated stellar image to the total flux,

$F R_{\text {obs }}=F_{\mathrm{FWHM}} / F_{\text {total }}$,

may be determined by creating curves of growth for individual stars (Stetson 1990). The larger number of nearly isolated stars found on the $H$-band image (used for PSF creation) allowed a reliable determination of $F R_{\text {obs }}$ only in $H$, although many stars on the $H$-band image were still too influenced by neighbours to study the aperture curve of growth in detail. We were able to create well-behaved curves of growth for 7 stars. As in the PSF fitting routine, we have used the FWHM of the PSF as kernel radius and as the reference flux for the flux ratio determination. These ratios range from 0.47 to 0.58 , with an average of $0.53 \pm 0.05$, i.e., $\sim 50 \%$ of the integrated point source flux are used for PSF fitting. In addition, the variation of the flux ratio $F R_{\text {obs }}$ over the field serves as an indicator of the Strehl ratio variation. If the AO correction mechanism is the dominant factor determining the concentration of the flux into the $F W H M$ kernel, the Strehl ratio and thus $F R_{\text {obs }}$ should decrease with distance form the guide star, as the seeing 
correction worsens. No correlation of the flux ratio with distance from the guide star is found. Though these are small number statistics, this supports the suggestion that the sensitivity variations over the field are not predominantly due to increasing distance from the guide star (Sect. 2.1.4).

\subsubsection{Photometric uncertainties and incompleteness calculation}

For the incompleteness correction, artificial frames were created with randomly positioned artificial stars. Magnitudes were also assigned automatically in a random way. Due to the very crowded field, only 40 stars were added to each artificial frame in order to avoid significant changes in the stellar density. A total of 100 frames was created for both the $H$ and $K^{\prime}$ deep exposures, leading to a total of 4000 artificial stars used in the statistics. In addition to the individual incompleteness in each band, the loss of sources due to scatter of the main sequence generated by the more uncertain photometry in the dense parts of the cluster was estimated. For this purpose the artificial $K^{\prime}$ stars were assigned a formal instrumental "colour" of $\left(H-K^{\prime}\right)=0.33 \mathrm{mag}$ (corresponding to $1.745 \mathrm{mag}$ after photometric transformation), derived from the average observed instrumental colour of the main sequence, and via this transformed into instrumental $H$ magnitudes. Artificial stars were inserted at the same positions in the $H$ and $K^{\prime}$ frames. In this way the procedure also accounts for stars lost due to the matching of $H$ and $K^{\prime}$ data. The artificial stars were calibrated using the colour equations shown in Sect. 2.1.3, thus allowing us to estimate the loss of stars in the mass function derivation due to the applied main sequence colour selection (see Sect. 5). This resulted in significantly larger corrections as compared to the individual filter recoverage without matching and colour selection. As an example, the results for the mass function calculation performed on the artificial stars are displayed in Fig. 4.

As the recovery rate depends strongly on the stellar density and thus radial distance from the cluster center, the incompleteness correction will be determined in dependence of the radial bin analysed when radial variations in the MF are studied (Sect. 5.3).

In addition to luminosity and mass function corrections, the artificial star tests were used to estimate the real photometric uncertainties by comparing inserted to recovered magnitudes of the artificial stars. The median difference between the original and the recovered magnitude of the artificial stars, $\Delta m_{\text {arti }}=<m_{\text {added }}-m_{\text {DAOPHOT }}>$, has been used as an estimate of the real photometric uncertainty. To obtain the median uncertainty, the intervalls $0<\Delta m_{\text {arti }}<1$ and $-1<\Delta m_{\text {arti }}<0$ have been treated individually, and the mean of the absolute value of both median values, weighted with the number of objects in each intervall, is quoted in Table 2. The overall flux deviation, including positive and negative deviations, is close to zero for stars brighter than $20 \mathrm{mag}(< \pm 0.004)$, and for fainter stars becomes -0.17 and -0.13 in $H$ and $K^{\prime}$, respectively, showing a tendency to underestimate the flux of faint stars. This tendency is more serious in the cluster center, where comparably large

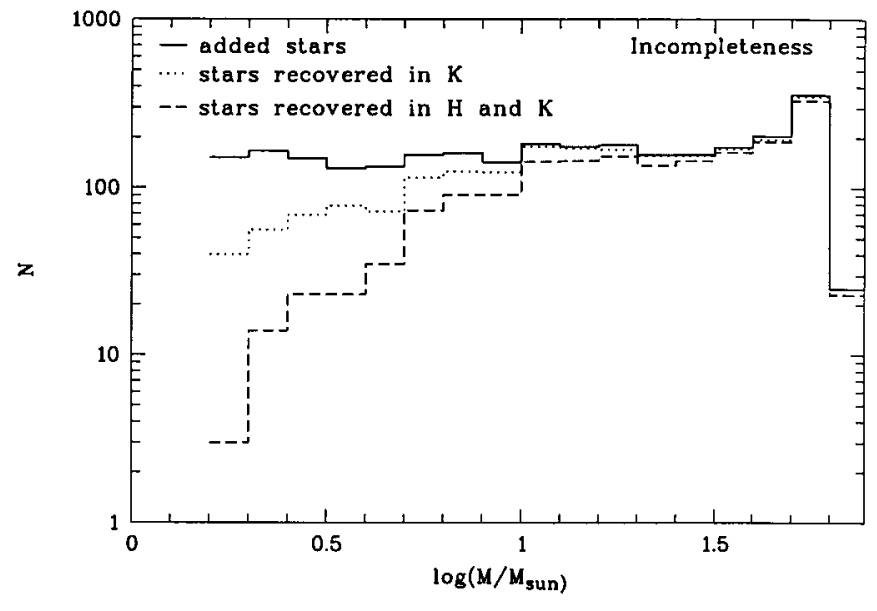

Fig. 4. Incompleteness tests performed on the Gemini $H$ and $K^{\prime}$ data. The plot demonstrates the necessity to include the matching between single filter observations to obtain a realistic estimate on the incompleteness, when matching of objects is needed to create colourmagnitude- and two-colour-diagrams.

uncertainties are already observed for magnitudes fainter than $18 \mathrm{mag}$ in both pathbands.

In a second test, photometry of the bright stars in the short exposures was compared to the magnitudes of the deep exposures, yielding $\Delta m_{\mathrm{sl}}=<m_{\text {short }}-m_{\text {long }}>$ using the same procedure as for artificial stars. Note, however, that the quality of the short exposures is much worse than the one of the long exposures due to the high background noise. Therefore, the artificial star experiments, for which only the deep exposures have been used, yield a more realistic estimate of the photometric uncertainties. The results of both tests are summarised in Table 2 .

The resulting uncertainty is roughly a factor of 2 to 3 larger than the theoretical magnitude uncertainty $\sigma_{\text {DAO }}$ determined from detector characteristics by DAOPHOT. The photometry in the cluster center shows a larger uncertainty than the photometry in the outskirts. As expected in a crowding-limited field, this also implies a reduced detection probability of faint sources in the center of the cluster.

\subsection{HST/NICMOS data}

HST/NICMOS observations have been obtained in the three broad-band filters $F 110 W, F 160 W$ and $F 205 W$, roughly equivalent to $J, H$ and $K$. The basic parameters are included in Table 1. For a detailed description of the HST data and their reduction see Figer et al. (1999).

\section{Photometric results}

\subsection{Radial colour gradient}

During the process of calibration we realised that a strong colour gradient is present in the Arches field. In Fig. 5, the $H-K^{\prime}$ colour is plotted against radial distance from the cluster center. As the extinction law has not yet been derived for HST filters, we have used the colour transformations in BGB to transform NICMOS into 2MASS magnitudes. Though the 
Table 2. Photometric uncertainties derived from artificial star experiments $\left(\Delta m_{\mathrm{arti}}\right)$ and the comparison of short and long exposures $\left(\Delta m_{\mathrm{sl}}\right)$. The photometric uncertainties determined by DAOPHOT are given for comparison. Note that for the brightest bin, 10-12 mag, only the photometry of the short exposures was available. The higher starting bin in $H$ is due to the calibration procedure of the inserted artificial stars. Magnitudes are given in the NICMOS system $(F 160 W, F 205 W)$.

\begin{tabular}{ccccccccccc}
\hline \hline \multirow{2}{*}{ Band } & mag & $\Delta m_{\text {arti }}$ & $\Delta m_{\text {sl }}$ & $\sigma_{\text {DAO }}$ & $\Delta m_{\text {arti }}$ & $\Delta m_{\text {sl }}$ & $\sigma_{\text {DAO }}$ & $\Delta m_{\text {arti }}$ & $\Delta m_{\text {sl }}$ & $\sigma_{\text {DAO }}$ \\
\hline \multicolumn{1}{c}{ all } & \multicolumn{2}{c}{$R<10^{\prime \prime}$} & \multicolumn{2}{c}{$R>10^{\prime \prime}$} \\
& $12-14$ & 0.007 & 0.059 & 0.004 & 0.012 & 0.052 & 0.005 & 0.004 & - & 0.002 \\
& $14-16$ & 0.015 & 0.061 & 0.008 & 0.025 & 0.065 & 0.010 & 0.011 & 0.035 & 0.004 \\
& $16-18$ & 0.044 & 0.128 & 0.015 & 0.077 & 0.120 & 0.017 & 0.038 & 0.134 & 0.009 \\
& $18-20$ & 0.119 & - & 0.036 & 0.167 & - & 0.039 & 0.098 & - & 0.030 \\
& $>20$ & 0.264 & - & 0.142 & 0.514 & - & 0.142 & 0.265 & - & 0.144 \\
$K^{\prime}$ & $10-12$ & 0.004 & - & 0.005 & 0.004 & - & 0.005 & 0.003 & - & 0.006 \\
& $12-14$ & 0.008 & 0.042 & 0.005 & 0.011 & 0.048 & 0.005 & 0.007 & 0.028 & 0.005 \\
& $14-16$ & 0.027 & 0.072 & 0.007 & 0.041 & 0.086 & 0.007 & 0.020 & 0.042 & 0.006 \\
& $16-18$ & 0.071 & 0.121 & 0.016 & 0.125 & 0.166 & 0.017 & 0.057 & 0.088 & 0.016 \\
& $18-20$ & 0.206 & - & 0.060 & 0.280 & - & 0.073 & 0.189 & - & 0.056 \\
& $>20$ & 0.411 & - & 0.274 & 0.520 & - & - & 0.360 & - & 0.274 \\
\hline
\end{tabular}

2MASS filters deviate slightly from the standard Johnson $J H K$ filters used to determine the extinction law (Rieke \& Lebofsky 1985), we will be able to estimate the approximate amount of change in visual extinction across the field. The extinction parameters from Rieke \& Lebofsky (1985) are given by

$A_{J} / A_{V}=0.282 \quad A_{H} / A_{V}=0.175 \quad A_{K} / A_{V}=0.112$,

where $A_{\text {Filter }}$ is the extinction in the given filter. For a change in extinction of $\Delta A_{V}$ this leads to

$$
\begin{aligned}
\Delta A_{V} & =\Delta\left(A_{J}-A_{H}\right) / 0.107 \\
\Delta A_{V} & =\Delta\left(A_{H}-A_{K}\right) / 0.063 \\
\Delta A_{V} & =\Delta\left(A_{J}-A_{K}\right) / 0.170 .
\end{aligned}
$$

We are able to measure the right-hand side of each expression from the transformed HST/NICMOS $J H K_{\mathrm{S}}$ photometry. The resulting $\Delta A_{V}$ is given in each plot in Fig. 6, together with the fitting uncertainty from the rms scatter in the colour.

From the linear fits in Fig. 6 we see that $A_{V}$ increases by about one order of magnitude over the entire field when moving outwards from the cluster center. The effect is most pronounced in the HST $J-K_{\mathrm{s}}$ vs. radius diagram (Fig. 6, bottom), where the longest colour baseline is used. We derive a change in visual extinction of $\Delta A_{V}=10.71 \pm 2.47 \mathrm{mag}$ over the Gemini field (1000 pixels $\hat{=} 0.8 \mathrm{pc}$ ). Notably, if only the innermost $5^{\prime \prime}$ ( 250 pixels, $0.2 \mathrm{pc}$ ) are fitted, no variation in $A_{V}$ is observed. When fitting the core separately, we get $\Delta A_{V}=0.77 \pm 1.12$ mag for $R<5^{\prime \prime}$ ( 250 pixels) versus $\Delta A_{V}=7.87 \pm 2.85 \mathrm{mag}$ for $5^{\prime \prime}<R<20^{\prime \prime}$ ( $250<R<1000$ pixels). The latter value corresponds to $\Delta A_{V}=10.5 \mathrm{mag} / 1000 \mathrm{pix}^{-1}$, consistent with the trend over the entire field. The small radial trend and low extinction value in the cluster center indicates the local depletion of dust. This could be either due to winds from massive stars or due to photo-evaporation of dust grains caused by the intense UV-radiation field.

A change in $A_{V}$ of $\sim 10 \mathrm{mag} / 1000 \mathrm{pix}^{-1}$ is also consistent with the result found in $J-H, 8.8 \pm 2.1 \mathrm{mag} / 1000 \mathrm{pix}^{-1}$, while a larger value of $\Delta A_{V}=14.9 \pm 3.2 \mathrm{mag}$ is derived from the $H-$ $K_{\mathrm{s}}$ plot. Due to the uncertainties we conclude that the extinction varies by $\Delta A_{V} \sim 9-15 \mathrm{mag}$ across the Arches field of $20^{\prime \prime}$ or $0.8 \mathrm{pc}$, most likely closer to the lower value. This is in any case a tremendous change in the dust column density along the line of sight, with strong implications on limiting magnitudes and the potential detection of faint objects.

We have used a linear fit to the colour variation with radius for $R>5^{\prime \prime}$ to correct for the strong change in reddening observed in the outer cluster field. The values for cluster center stars $\left(R<5^{\prime \prime}\right)$ have been left unchanged, due to the large scatter and the very small trend found. Thus, these adjusted colours are scaled to the cluster center, where $A_{V}$ is lowest. From the Rieke \& Lebofsky (1985) extinction law, the change in $K$-band magnitude with radius corresponding to the change in colour can be derived as $\Delta A_{K}=0.112 / 0.063 \cdot \Delta A_{H-K}$. We have used this relation to adjust the $K$-band magnitudes accordingly. The "dereddened" colour-magnitude diagrams will be shown in direct comparison with the observed CMDs in Sect. 3.3 (cf. Fig. 9).

\subsection{Extinction maps}

We can calculate the individual extinction for each object by shifting it along the reddening vector onto the isochrone. If we assume that intrinsic reddening plays only a minor role for most of the stars within the cluster, we can combine the individual reddenings to estimate the spatial extinction variation. In practice, we have applied a reddening of $A_{V}=15 \mathrm{mag}$ to a $2 \mathrm{Myr}$ isochrone of the Geneva set of models (Lejeune $\&$ Schaerer 2001). This choice of reddening ensures that the isochrone serves as a blue envelope for the bulk of the cluster stars, which are significantly more reddened. The choice of the isochrone will be discussed in context with the mass function (Sect. 5), where the physical effects of the population model used are more important. As discussed in Grebel et al. (1996), colours of main-sequence stars also depend on binarity, stellar 


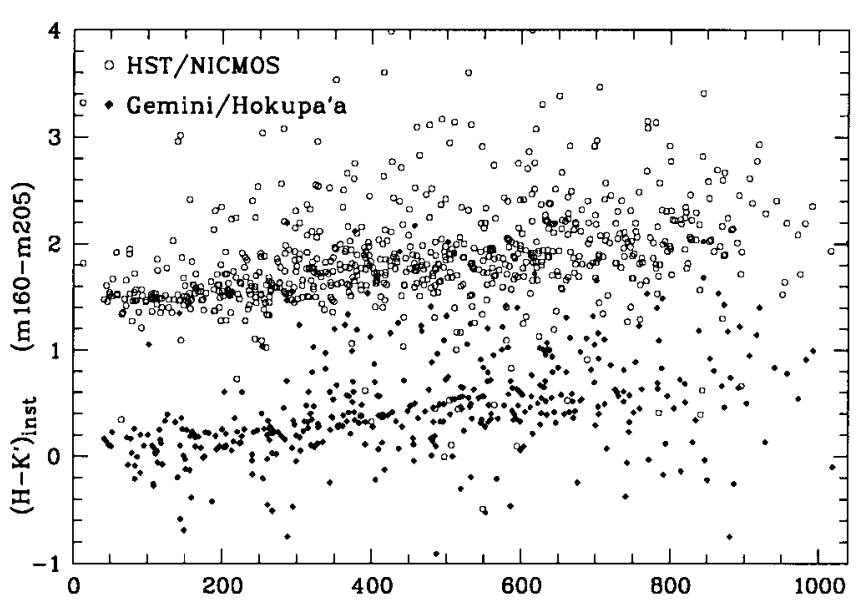

Fig. 5. Colour variation across the Gemini field, as observed in instrumental Gemini magnitudes and HST/NICMOS data. As a trend of increasing colour excess with increasing distance from the Arches cluster center is observed in the two independent datasets, an instrumental effect as the cause for this variation is highly improbable.

rotation, and intrinsic infrared excess. We ignore these effects here since we have no means to distinguish them from reddening effects. In the worst case, we will overestimate the reddening for some stars. We have then shifted the stars along the reddening path in colour-magnitude space, again assuming that the relative slopes of the extinction law approximately follow a Rieke \& Lebofsky (1985) law. The resulting extinction map for the Gemini photometry is shown in Fig. 7.

Since the original HST/NICMOS field has twice the area of the Gemini central Arches field, we have also calculated the extinction map for the entire NICMOS field following the same procedure. The corresponding map is shown in Fig. 8.

The extinction measured with this procedure in the $K$ band lies in the range $1.9<A_{K}<4.1 \mathrm{mag}$, with an average value of $3.1 \mathrm{mag}$, corresponding to $16<A_{V}<37 \mathrm{mag}$, $\left.<A_{V}\right\rangle=27.7 \mathrm{mag}$. Cotera et al. (2000) derive a near-infrared extinction of $2.8<A_{K}<4.2 \mathrm{mag}$, with an average value of $\left\langle A_{K}\right\rangle=3.3 \mathrm{mag}$ for 15 lines of sight towards several Galactic Center regions, corresponding to an average visual extinction of $\left\langle A_{V}\right\rangle=29.5$ mag (transformed using Rieke \& Lebofsky 1985). They obtain the highest extinction towards a field close to the Arches cluster, $A_{V}=37.5 \mathrm{mag}$. This is very close to our highest extinction value. The average value determined from individual dereddening here is the same as the average extinction obtained by Figer et al. (1999), $\left\langle A_{V}\right\rangle=27.7$ mag. Note that the typical random scatter $\sigma\left(A_{K}\right)$ from foreground dust density fluctuations found in GC fields is linearly related to the average extinction within a field. The relation determined by Frogel et al. (1999) from giant branch stars in 22 pointings towards fields within $4^{\circ}$ from the $\mathrm{GC}$ is given by $\sigma\left(A_{K}\right)=0.056( \pm 0.005)<A_{K}>+0.043( \pm 0.005)$. This yields an expected natural scatter from GC clouds of only $\sigma\left(A_{K}\right)=0.22$ mag for $\left\langle A_{K}\right\rangle=3.1 \mathrm{mag}$, much below the difference in reddening observed in the Arches field. Thus, the change in extinction cannot be explained by the natural fluctuations of the dust distribution in the $\mathrm{GC}$ region.
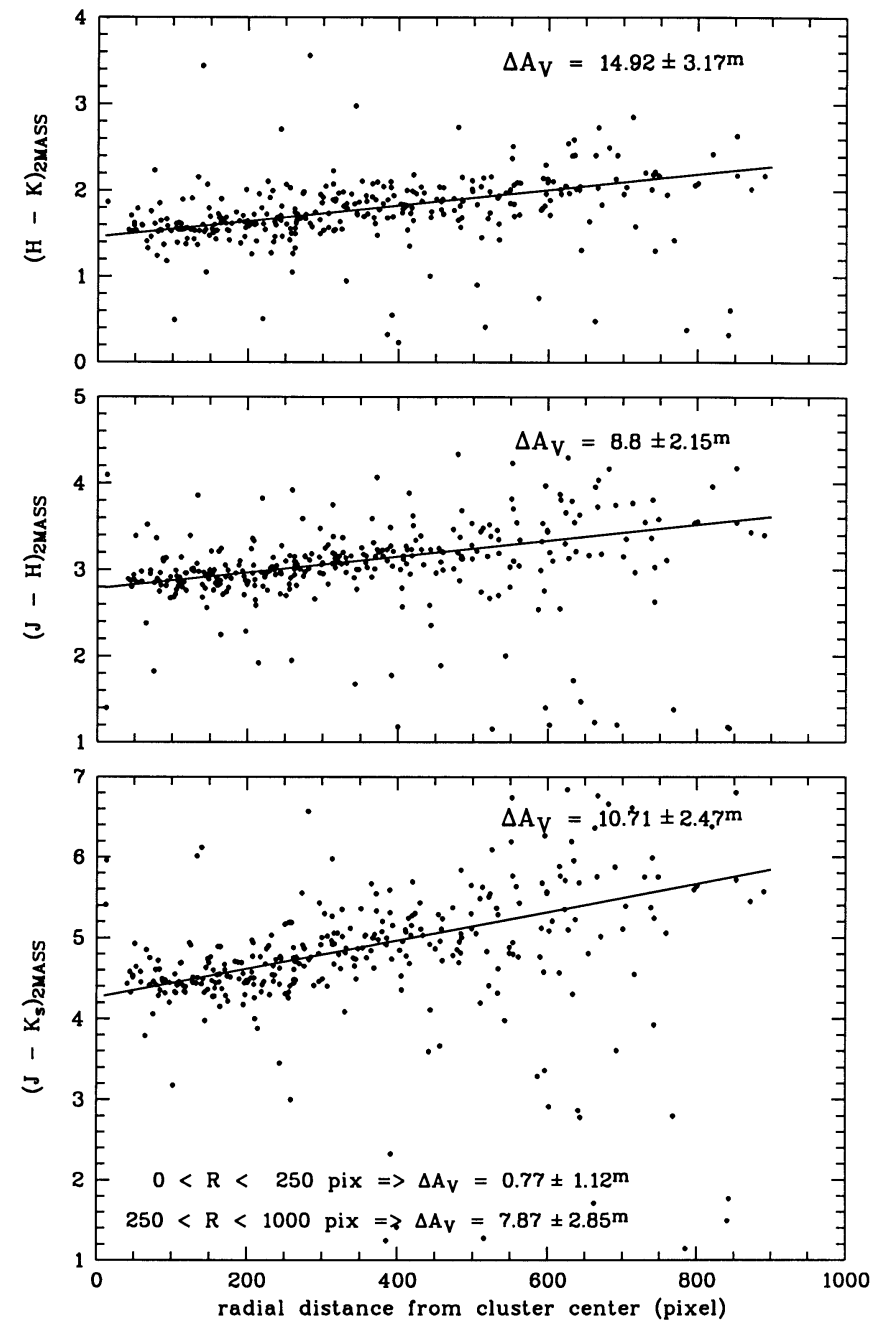

Fig. 6. Colour trends over the Arches field as observed in the HST/NICMOS data set within the area covered by the Gemini observations. The radial distance from the cluster center is given in pixels on the Gemini scale. For the calculation of $\Delta A_{V}$, the Rieke \& Lebofsky (1985) reddening law has been assumed.

Comparison of the cluster center main sequence population with the main sequence colour of a theoretical $2 \mathrm{Myr}$ isochrone from the Geneva set of models (Lejeune \& Schaerer 2001), later-on used for the derivation of the mass function, yields an average extinction of $A_{V}=24.1 \pm 0.8 \mathrm{mag}$ in the cluster center. This extinction value has been used to transform isochrone magnitudes and colours into the cluster magnitude system. It has been suggested that the brightest and most massive stars in Arches are Wolf-Rayet stars of type WN7 (Cotera et al. 1996; Blum et al. 2001). Fundamental parameters of Wolf-Rayet stars are compiled in Crowther et al. (1995). For stars of subtype WN7 they find typical colours of $(H-K) \sim 0.2 \mathrm{mag}$, leading to an extinction of $A_{V}=24.9 \pm 2.4$ mag with an observed $H-K$ colour of $\sim 1.77$ mag for the WN7 stars, which were identified by comparison with the Blum et al. (2001) narrow band photometry. This value is in very good agreement with the $A_{V}$ determined from the main sequence colour in the cluster center. 

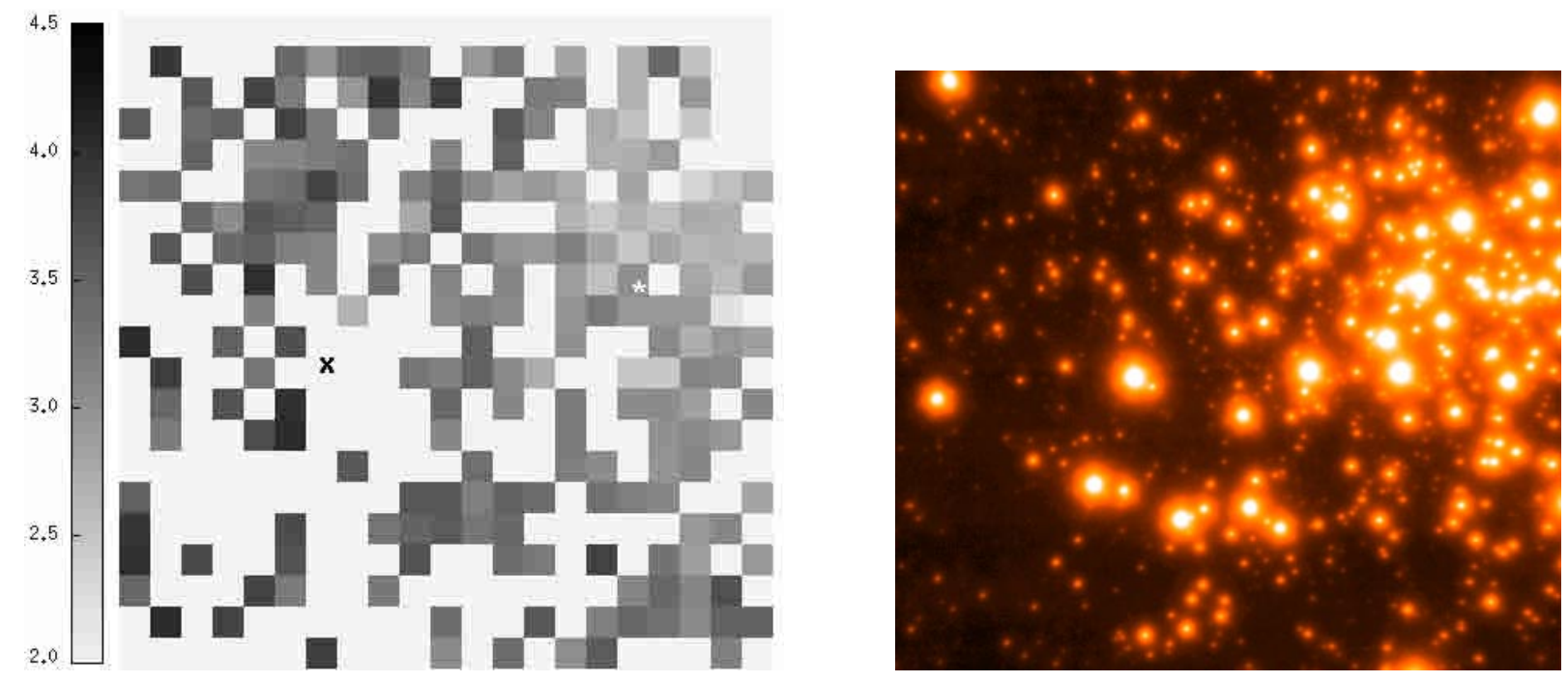

Fig. 7. $A_{K}$ extinction map, binned in the same manner as the residual map in Fig. 3 (North is up, East is to the right). White spots are positions without stars for evaluation. The individual extinction has been calculated by shifting the stars in the $K$ vs. $H-K$ colour-magnitude diagram to a $2 \mathrm{Myr}$ isochrone offset bluewards of the main sequence. Transformation to $A_{K}=0$ mag has been performed afterwards, to avoid large errors in the shifting procedure. This results in a minimum $A_{K}$ of $1.86 \mathrm{mag}$, and a maximum of 4.08 mag, assuming a Rieke \& Lebofsky (1985) extinction law.
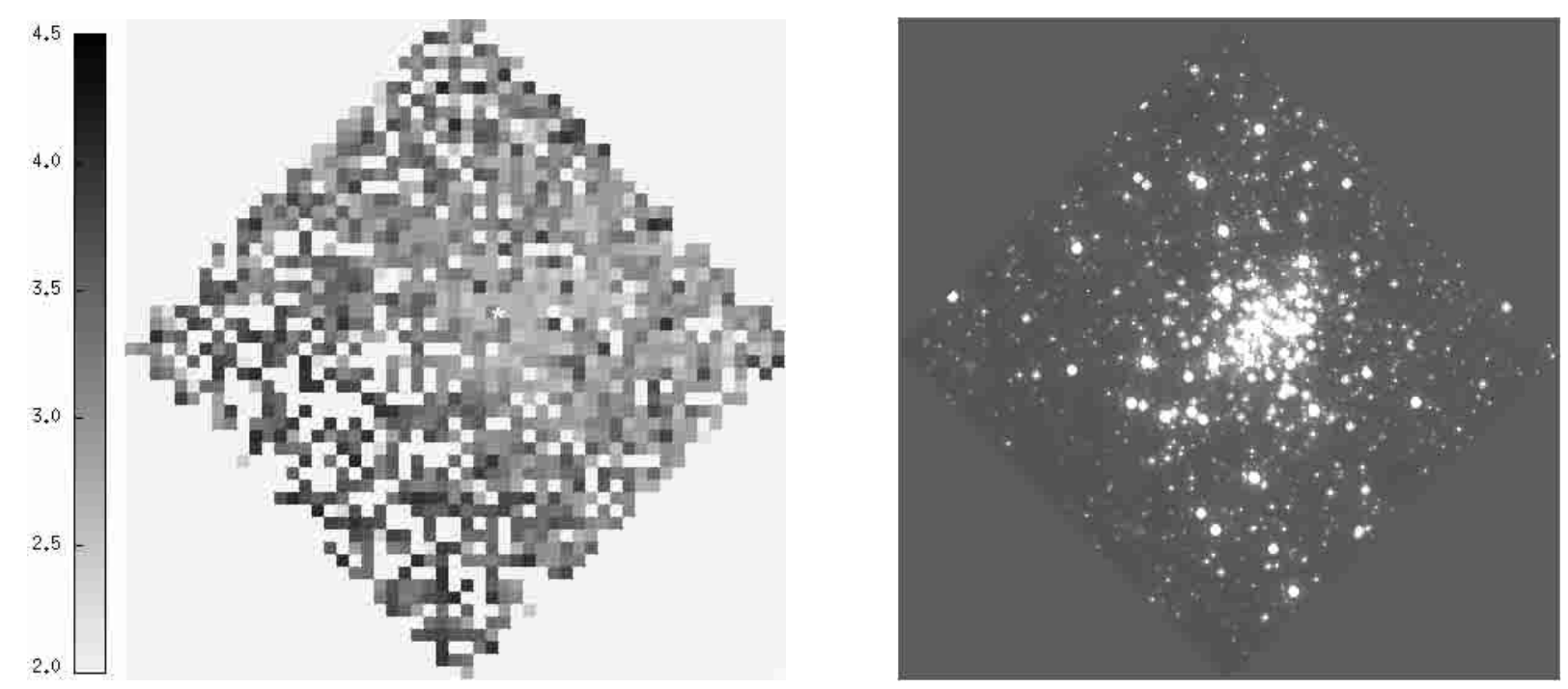

Fig. 8. $A_{K}$ extinction map derived from HST $m 205$ photometry. See Fig. 7 for details. The coordinate transformed HST/NICMOS $F 205 W$ image is also shown for comparison. Note the different scales (HST/NICMOS: 40" $\times 40^{\prime \prime}$, Gemini/Hokupa'a: $\left.20^{\prime \prime} \times 20^{\prime \prime}\right)$.

\subsection{Colour-magnitude diagrams}

The resulting colour-magnitude diagrams for Gemini and HST are presented in Fig. 9 (upper panel). Two important differences are seen when inspecting the CMDs. First, the scatter in the main sequence is significantly larger in the ground-based photometry. While the HST/NICMOS CMD reveals a narrow main sequence in the cluster center (circles in Fig. 9), the same stars display a much larger colour range in the Gemini CMD. The poor Strehl ratio in the Gemini/Hokupa'a data as compared to the HST/NICMOS data (see Sect. 2.1.5) causes a high, nonuniform additional background due to uncompensated seeing halos around bright stars, which decreases photometric accuracy. In the dense regions of the cluster center, where crowding problems are most severe, the photometry is most affected.
The number of faint, unresolved companion stars that merge into the high stellar background underneath the bright cluster population is very high. As discussed in Sect. 2.1.4, the halos of the bright stars hinder the detection of faint objects despite the principally high spatial resolution seen in individual PSF kernels. Operating at the diffraction limit, NICMOS is not restricted by these effects, yielding a better effective resolution especially in the dense regions. A tighter main sequence and less scatter is the consequence. In Fig. 9, the innermost 5" of the Arches cluster are marked by open circles. It is clearly seen that most massive (bright) stars are located in the cluster center.

A second effect observed is the much larger number of faint objects seen in the HST data (cf. Fig. 2). As the limiting magnitude and the measured spatial resolution of the images are 

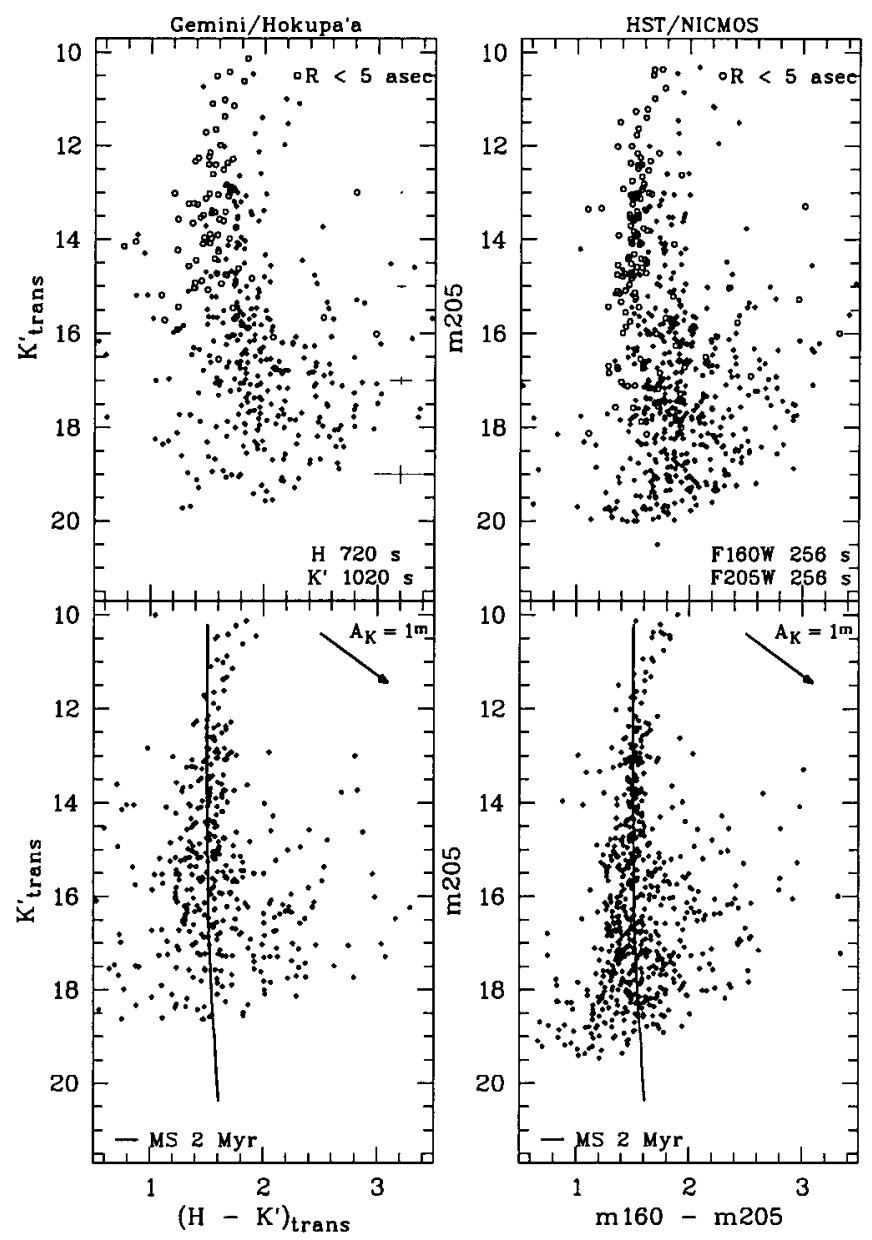

Fig. 9. Colour-magnitude diagrams. Left: Gemini/Hokupa'a, right: HST/NICMOS. Lower panel: CMDs corrected for radial reddening gradient (Sect. 3.1).

similar in both datasets, this, too, has to be a consequence of the low Strehl ratio in the AO data.

The lower panel of Fig. 9 shows the "dereddened" CMDs, corrected for the radial colour gradient found and the corresponding change in extinction, $\Delta A_{K}$ (Sect. 3.1). The colours of stars beyond $R>5^{\prime \prime}$ have been adjusted to the colour of the cluster center. Comparison with the original CMDs shows that most of the bright, seemingly reddened stars fall onto the same main sequence after correcting for the colour trend. These stars are located at larger distances from the cluster center and thus suffer from more reddening by residual dust. As will be discussed in the context of the mass function (Sect. 5), these stars might have formed close to the cluster at a similar time as the cluster population. At the faint end of the CMD, there are, however, a large number of objects that remain unusually red after the correction has been applied. These objects may either be pre-main sequence stars or faint background sources. Unfortunately, we are not able to disentangle these two possible contributions, and will thus exclude objects significantly reddened relative to the main sequence when deriving the mass function.

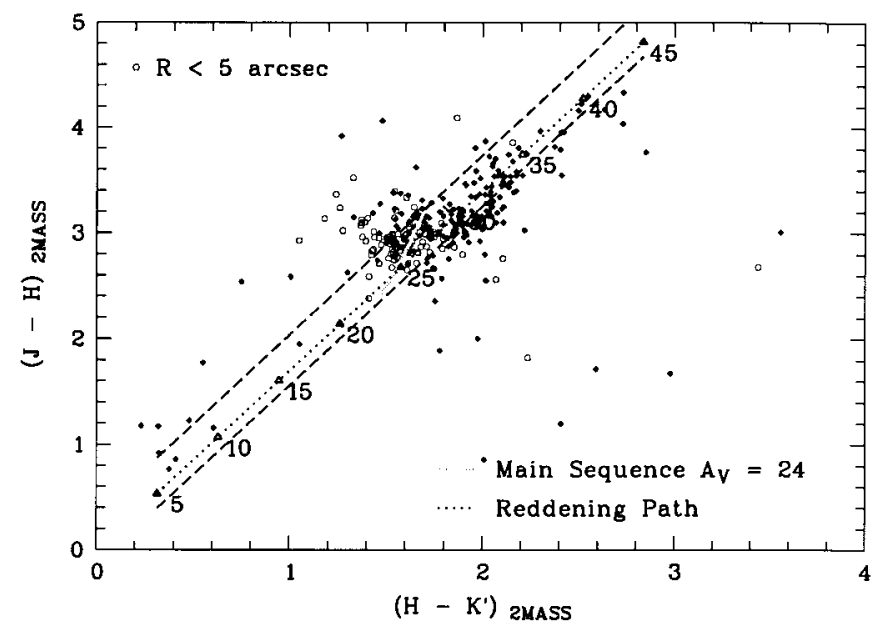

Fig. 10. Two-colour diagram from HST observations. The reddening path is shown as a straight line labeled with $A_{V}$ values, and the main sequence is indicated by the thick grey line. The area between the dashed lines marks the region of reddened main sequence stars.

\subsection{HST/NICMOS colour-colour diagram}

For comparison with the reddening path and a main sequence in standard colours, the NICMOS filters have been transformed into the 2MASS $J H K_{\mathrm{s}}$ system. In Fig. 10, we show the transformed HST/NICMOS colour-colour diagram for the stars bright enough to be observed in all three filters. The $A_{V}$ values are from the Rieke \& Lebofsky (1985) extinction law for standard JHK photometry. Though we are aware of the uncertainties inherent to the transformation of severely reddened stars, the proximity of the reddening path to the data points supports the validity of the equations derived by BGB. Changing the transformation parameters slightly results in a large angle between the data points and the reddening path.

A wide spread population of stars is clearly seen along the reddening path, as expected from the colour trend discussed in Sect. 3.1 (no correction for the varying extinction has been applied in this diagram). Again, the stars with the lowest reddening within the cluster population are the bright stars in the Arches cluster center. Moving along the reddening line towards higher values of $A_{V}$ mainly means moving radially outwards from the cluster center. As in the CMD, a correction for the observed colour gradient causes the bulk of the stars to fall onto the main sequence with a reddening of $A_{V}=24 \mathrm{mag}$, corresponding to the cluster center.

\section{Luminosity functions and incompleteness effects}

\subsection{Integrated luminosity function}

In Fig. 11, we compare the Gemini with the HST luminosity function for the $K_{\text {trans }}^{\prime}$ vs. $m 205$ observations. For direct comparison of the observational efficiency, no colour cut has been applied, but the entire physically reasonable colour range from approximately 0 to $4 \mathrm{mag}$ in $H-K^{\prime}$, including reddened and foreground objects, has been included in the luminosity function (LF). Therefore, these LFs are not the ones from which 


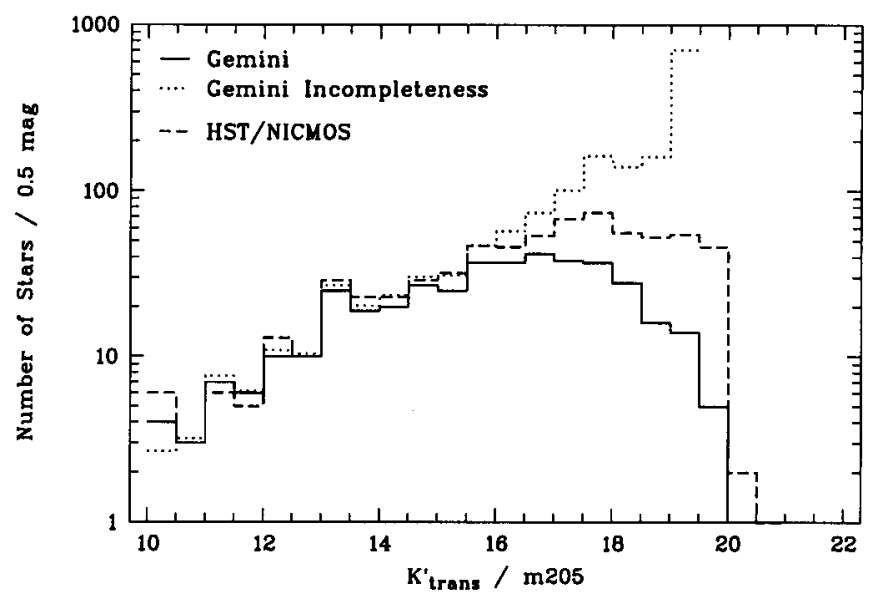

Fig. 11. Comparison of Gemini versus HST luminosity functions.

the mass functions have been derived. The only selection criterion that has been applied is a restriction of the photometric uncertainty in both magnitude $\left(\sigma_{K^{\prime}}<0.2 \mathrm{mag}\right)$ and colour $\left(\sigma_{H-K^{\prime}}<0.28 \mathrm{mag}\right.$, corresponding to $\sigma_{K^{\prime}}<0.2 \mathrm{mag}$ and $\left.\sigma_{H}<0.2 \mathrm{mag}\right)$. The uncertainty selection in colour allowed us to select only those objects that have been detected with high confidence in both $H$ and $K^{\prime}$ images in the Gemini data, and in the $F 160 W$ and $F 205 W$ filters in the NICMOS data, respectively. The colour-uncertainty selection on the HST data simulates the matching of $H$ and $K^{\prime}$ detections used on the Gemini data for the selection of real objects. Thus, only objects that are detected in both $H$ and $K^{\prime}$ have been included in the luminosity function. This gives us some confidence that we are not picking up hot pixels or cosmic ray events. The area covered with Gemini has been selected from the HST photometry as displayed in Fig. 1.

As can be seen in Fig. 11, many objects are missed by Gemini in the fainter regime, though the actual limiting (i.e., cut-off) magnitudes are the same in both datasets. This is due to the fact that $50 \%$ of the light is distributed into a halo around each star. These halos prevent the detection of faint objects around bright sources, especially in the crowded regions. This effect is most obvious when examining the starsubtracted frames resulting from the DAOPHOT allstar task. In these frames the cluster center is marked by a diffuse background, enhanced by $\sim 20$ counts in $K^{\prime}$ and $\sim 40$ counts in $H$ above the observational background of 2 and 4 counts in the cluster vicinity, respectively. In addition to the simple crowding problem due to the stellar density affecting both datasets, the overlap of many stellar halos hinders the detection of faint stars in the Gemini data. At larger radial distances from the cluster center, more and more faint stars are detected both in the HST as well as in the Gemini data (Fig. 12).

The fact that the incompleteness corrected Gemini LFs follow closely the shape of the HST LFs supports the results of our incompleteness calculations, which will be used to determine the incompleteness in the mass function.

\subsection{Radial variation of the luminosity function}

Radial luminosity functions were calculated in $\Delta R=5^{\prime \prime}$ bins, using the same uncertainty selection as in Fig. 11 (Sect. 4.1). The resulting radial LFs are shown in Fig. 12, along with the incompleteness determined for each radial bin. In the cluster center (lowest panel), the very good match of the Gemini and HST LFs for $K_{\text {trans }}^{\prime}<16$ mag reveals the comparable spatial resolution obtained in both data sets. Despite the strong crowding seen already in these bright stars, the Gemini AO data resolve the sources in the cluster center nicely. When we move on to fainter magnitudes, however, we are limited by the halos of these bright stars, as discussed above. The clear decrease below $K_{\text {trans }}^{\prime}=16$ mag marks the point where stars are lost due to the enhanced background. When we move radially outwards, the limiting magnitude above which faint stars are lost shifts towards fainter magnitudes. The tendency to loose the faint tail of the magnitude distribution nevertheless remains clearly seen, though it becomes much less pronounced for $R>10^{\prime \prime}$, where the Gemini and HST LFs resemble each other. For $R>15^{\prime \prime}$ (upper panel) we are limited by small number statistics due to the small area in this radial bin. As it is hard to observe a welldefined LF at these radii, we will add the two upper bins when we create the radially dependent mass functions in Sect. 5.3.

The magnitude-dependent distribution of stars within the cluster is evident in these LFs. While bright stars are predominantly found in the cluster center, their number density strongly decreases with increasing radius. When we analyse the Gemini LFs more quantitatively, we find 25 (50) stars with $K_{\text {trans }}^{\prime}<13(14)$ mag within $R<5^{\prime \prime}$, but only 8 (23) such stars with $5<R<10^{\prime \prime}$, and beyond 10", we observe only 7 (11) such stars. The numbers for HST are comparable in the bright magnitude bins. On the other hand, the number of faint stars with $K_{\text {trans }}^{\prime}>18(19)$ mag increases from 1 (0) to 14 (3) to 48 (16). As we see significantly more faint stars in the HST data, the corresponding numbers are higher, i.e. the number of stars with $m 205>19$ mag is 0 in the innermost bin, 24 in the intermediate bin, and 81 in the outermost bin. Despite the fact that the area on the Gemini frame increases by about a factor of 3 between the inner and intermediate bin, the number of bright stars is strongly diminished beyond a few arcseconds, while the number of the faint stars increases by much more than the change in area can account for. Although for the fainter stars the effects of crowding and a real increase in the fainter population of the cluster cannot be disentangled, the decrease in the number of bright stars is a clear indication of mass segregation within the Arches cluster.

\section{Mass function}

The mass function (MF) may be defined as the number of stars observed in a certain mass bin. The mass function in stellar populations is most frequently fitted by a power law, whose slope depends on the mass range analysed (e.g., Kroupa 2001). In the logarithmic representation, the mass function is defined as

$\mathrm{d}(\log N) / \mathrm{d}(\log M) \sim M^{\Gamma}$ 


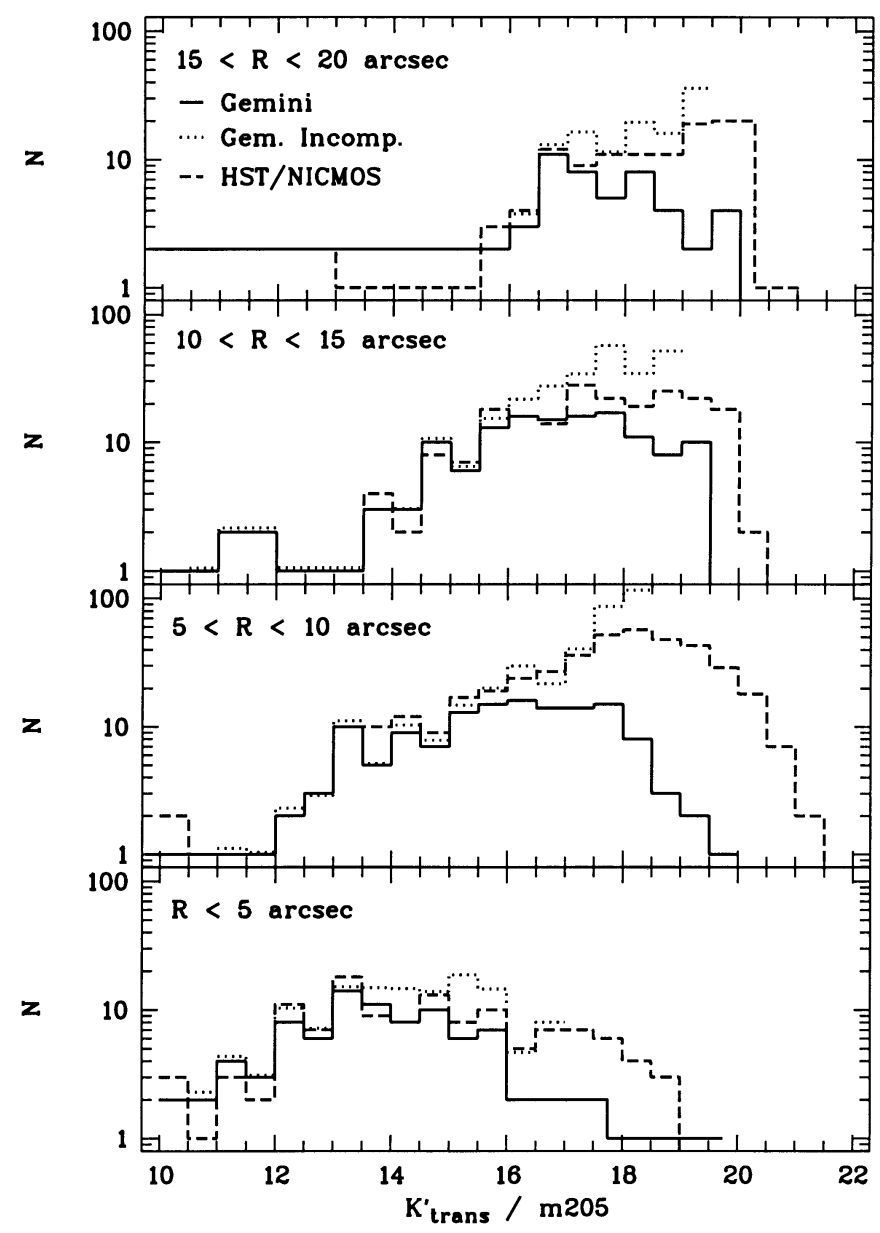

Fig. 12. Radial variation of the luminosity function. The comparison of Gemini and HST LFs is shown together with the Gemini incompleteness calculation. The dependence of the magnitude limits on the distance to the cluster center is striking.

where $M$ is usually given in solar masses, and $\Gamma$ is the slope of the mass function. The exact shape of the mass function and, in particular, its slope, has been subject of intense discussion (see, e.g., Massey 1995a, 1995b; Scalo 1986; and Scalo 1998 for a review). Massey et al. (1995a, 1995b) report slopes between -0.7 and -1.7 with a weighted mean of $-1.1 \pm 0.1$ for $M>7 M_{\odot}$ in several starforming regions in the Milky Way, and $-1.1<\Gamma<-1.6$ with a mean of $-1.3 \pm 0.1$ for $M>25 M_{\odot}$ in the LMC. From these studies in young clusters and associations, the mass function has been suspected to be rather universal, following a Salpeter (1955) power law with a slope of $\Gamma=-1.35$ for stars with masses $M \geq 1 M_{\odot}$ (Kroupa 2001). For the two starburst clusters studied in the Milky Way, Arches (FKM) and NGC 3603 (Eisenhauer et al. 1998), a flat slope of $\sim-0.7$ is observed for masses $M>10 M_{\odot}$ in Arches, and $M>1 M_{\odot}$ in NGC 3603 . These values have to be kept in mind as a comparison for the results presented in the following sections. The mass regime which will be discussed here spans a mass range of $6<M<65 M_{\odot}$ for Arches cluster stars.

\subsection{Integrated mass function}

The present-day mass function (Fig. 13) of the Arches cluster has been derived from the colour-magnitude diagram by transforming stellar luminosities into masses via a $2 \mathrm{Myr}$ isochrone from the Geneva basic set of stellar evolution models (Lejeune \& Schaerer 2001) using the method described in Grebel \& Chu (2000). Enhanced mass loss models were also used, but did not alter the resultant mass function. Stellar evolution (mass loss, giant evolution) is not important on timescales of the Arches age of $\sim 2 \mathrm{Myr}$ for stars with initial masses of $M<50 M_{\odot}$ $\left(\log \left(M / M_{\odot}\right)<1.7\right.$, i.e. stellar evolution affects the two upper mass bins of the MF at most). No attempt has been made to reconstruct the initial mass function (IMF) from the present-day MF for $M>50 M_{\odot}$. A distance modulus of $14.5 \mathrm{mag}$ and an extinction of $A_{V}=24.1 \mathrm{mag}$ have been applied.

The slopes of all mass functions discussed have been derived by performing a weighted least-squares fit to the number of stars per mass bin. The size of the mass bins was chosen to be $\delta \log \left(M / M_{\odot}\right)=0.1$ as the best compromise between mass function resolution and statistical relevance. This bin size is significantly larger than the photometric uncertainty in the considered mass and thus magnitude ranges. Only mass bins with a completeness factor of $\geq 75 \%$ have been included in the fit.

Note that we have not attempted to subtract the field star contribution. As can be seen in Fig. 1, the Gemini field is mostly restricted to the densest cluster region. When comparing to an arbitrary part of the GC field, we do not expect to observe the same distribution of stars as in the Arches field, as the faint, reddened background sources are negligible due to the high density of bright sources in the cluster area.

In addition, the stellar density in the GC is strongly variable, imposing additional uncertainties on the field contribution. Neither the Gemini nor the HST field covers enough area to estimate the field star population in the immediate vicinity of the cluster. A main sequence colour cut $(1.15<H-K<$ $1.90 \mathrm{mag}$ ) has been applied to the colour-corrected CMDs to select Arches members, excluding blue foreground and red background sources.

To allow for a direct comparison with the results obtained in FKM, we have used isochrones calculated for a metallicity of $Z=0.04$ for all MF derivations. The derived MFs are displayed in Fig. 13. The overall mass function derived from the Gemini data displays the same slope as derived from NICMOS within the uncertainties, namely $\Gamma_{\text {Gemini }}=-0.77 \pm 0.16$ and $\Gamma_{\mathrm{HST}}=-0.82 \pm 0.14$ fitted for $10<M<65 M_{\odot}$ (Fig. 13), which may be extrapolated down to $6 M_{\odot}$ when taking into account the incompleteness correction. The present-day upper mass of $65 M_{\odot}$ corresponds to an initial mass of about $100 M_{\odot}$ according to the Geneva models. FKM derive an overall slope of $\Gamma=-0.7 \pm 0.1$ in the inital mass range $6<M<120 M_{\odot}$, in good agreement with our results. The remaining difference in the maximum mass is due to the different extinction and the extinction corrections applied, which represent the largest uncertainties in the mass function derivation. As in particular the correction of the $K$ magnitude for the varying extinction is uncertain due to the unknown extinction law of the NICMOS filters, we have also derived the mass function for uncorrected $K$ 
magnitudes, with only the colour correction applied, which is independent of the extinction law assumed. In this case, the MF appears flatter with a slope of $\Gamma \sim-0.5 \pm 0.2$ (Fig. 13, lower panel). The discrepancy in the derived slopes clearly shows that the effects of differential extinction are not negligible, especially when deriving mass functions for very young regions, where the reddening varies significantly.

Furthermore, we have checked the effect of the binning on the MF by shifting the starting point of each bin by one tenth of the bin-width, $\delta \log \left(M / M_{\odot}\right)=0.01$. The resultant slopes range from $-0.69 \pm 0.13<\Gamma_{\text {Gemini }}<-0.90 \pm 0.15$ and $-0.79 \pm 0.12<$ $\Gamma_{\mathrm{HST}}<-0.98 \pm 0.13$. The average slopes for Gemini and HST, $\Gamma_{\text {Gemini }}=-0.77 \pm 0.15$ and $\Gamma_{\mathrm{HST}}=-0.86 \pm 0.13$, respectively, agree well within the errors. The slightly flatter slope observed in the Gemini data may reflect the more severe incompleteness due to crowding. Although all slopes are consistent within the errors, the range in slopes derived by scanning the bin-step shows that statistical effects due to the binning may not be entirely neglected in the MF derivation.

The metallicity within the immediate Galactic Center region has been a matter of discussion during the past decade. Several authors report supersolar metallicities derived from $\mathrm{CO}$ index strength and TiO bands in bulge stars (Frogel \& Whitford 1987; Rich 1988; Terndrup et al. 1990, 1991). Carr et al. (2000) measure $[\mathrm{Fe} / \mathrm{H}]=-0.02 \pm 0.13$ dex for the 7 Myr old supergiant IRS 7, and Ramirez et al. (2000) derive an average of $[\mathrm{Fe} / \mathrm{H}]=+0.12 \pm 0.22 \mathrm{dex}$ for 10 young to intermediate age supergiants, both very close to the solar value.

Using a 2 Myr isochrone with solar metallicity $Z=0.02$, the average slope of all bin steps is $\Gamma_{\text {Gemini }}=-0.84 \pm 0.13$ and $\Gamma_{\mathrm{HST}}=-0.91 \pm 0.12$. The mass function is thus not significantly altered when using solar instead of enhanced GC metallicity. We note, however, that a lower metallicity (i.e., in this case solar) steepens the MF slightly, thus working into the same direction as the incompleteness correction.

FKM report a flat portion of the MF in the range $15<M<$ $50 M_{\odot}$, which is not seen in the Gemini MF. This plateau can, however, be recovered, when we create a MF from $K$-band magnitudes uncorrected for differential extinction, and use a lowest mass of $\log \left(M_{\text {low }} / M_{\odot}\right)=0.25$. For $\log \left(M_{\text {low }} / M_{\odot}\right)=$ 0.20 the plateau is seen neither with nor without extinction correction. This, again, shows the dependence of the shape of the MF on the extinction corrections applied, as well as on the chosen binning.

From the considerations above, we conclude that the overall mass function of the Arches cluster has a slope of $\Gamma=-0.8$ to -0.9 in the range $6<M<65 M_{\odot}$. Although the uncertainty of missing lower mass stars in the immediate cluster center remains, the incompleteness correction strongly supports the derived shape of the MF. If the flat slope would be solely due to a low recovery rate of low-mass stars in the cluster center, this should be visible in a much steeper rise of the incompleteness corrected MF in contrast to the observed MF. We thus conclude that the slope of the MF observed in Arches is flatter than the Salpeter slope of $\Gamma=-1.35$, assumed to be a standard mass distribution in young star clusters. Such a flat mass function is a strong indication of the efficient production of high-mass stars in the Arches cluster and the GC environment.

\subsection{Effects of the chosen isochrone, bin size, and metallicity}

Blum et al. (2001) estimate a cluster age of 2-4.5 Myr for Arches assuming that the observed high-mass stars are of type WN7. If we compare the Geneva basic grid of isochrones with fundamental parameters obtained for WN7 stars (Crowther et al. 1995), a reasonable upper age limit for this set of isochrones is $\sim 3.5 \mathrm{Myr}$. Crowther compares the parameters derived for WN stars with evolutionary models at solar metallicity from Schaller et al. (1992) and with the mass-luminosity relation for O supergiants from Howarth \& Prinja (1989), yielding a mass range of $20-55 M_{\odot}$, but with high uncertainties at the low-mass end. The more reliable mass estimates for the colour and magnitude range observed for WN stars in Arches are restricted to $35<M<55 M_{\odot}$. From spectroscopic binaries, the masses of two WN7 stars are determined to be $\sim 30 M_{\odot}$ and $>48 M_{\odot}$ (Smith \& Maeder 1989). The theoretical lower limit to form Wolf-Rayet stars is $25 M_{\odot}$ for the Geneva models (Schaerer et al. 1993).

In the Geneva basic grid of models with $Z=0.04$, the 3.5 Myr isochrone is limited by a turnoff mass of $32 M_{\odot}$. We have thus calculated mass functions for isochrones with ages 2.5, 3.2, and 3.5 Myr in addition to the $2 \mathrm{Myr}$ case discussed above. Though the derived slopes scatter widely, irrespective of the isochrone used, the slope of the MF tends to be even flatter for any of the older population models. We thus conclude that, regardless of the choice of model and parameters, the Arches mass function displays a flat slope.

\subsection{Radial variation in the mass function}

The radial variation (Fig. 14) of the mass function is particularly interesting with respect to YC evolution. We have analysed the stellar population in Arches within three different radial bins, $0^{\prime \prime}<R<5^{\prime \prime}, 5^{\prime \prime}<R<10^{\prime \prime}$ and $10^{\prime \prime}<R<20^{\prime \prime}$. The resulting mass functions for the Gemini and HST datasets, along with the radius dependent incompleteness correction for the Gemini MFs, are displayed in Fig. 14.

We confirm the flat mass function observed by FKM in the innermost regions of the cluster, which steepens rapidly beyond the innermost few arcseconds. Most of the bright, massive stars are found in the dense cluster center, where the mass function slope is very flat. FKM derive a slope of $\Gamma=-0.1 \pm 0.2$ from the HST data in this region, which is consistent with Fig. 14. It is obvious from the lowest panel in Fig. 14 that we are crowding limited in the innermost region. We have thus not tried to fit a slope for $R<5^{\prime \prime}$.

In the next bin, $5^{\prime \prime}<R<10^{\prime \prime}$, the mass function obtained from the weighted least-squares fit has already steepened to a slope of $\Gamma=-1.0 \pm 0.3$. Again, the MF in this radial bin remains significantly flatter $(\Gamma=-0.5 \pm 0.4)$ when no $A_{K}$-correction is applied. Beyond $10^{\prime \prime}(0.4 \mathrm{pc}$, upper panel), a power law can only be defined in the range $10<M<30 M_{\odot}$ $\left(1.0<\log \left(M / M_{\odot}\right)<1.5\right)$, where a slope of $\Gamma=-1.69 \pm 0.66$ is found, consistent with a Salpeter $(\Gamma=-1.35)$ law. The large error obviously reflects the small number of mass bins used in the fit. Nevertheless, Fig. 14 clearly reveals the steepening of the MF very soon beyond the cluster center. 
For a more quantitative confirmation of the mass segregation present in the Arches cluster, we created cumulative functions for the mass distributions in the three radial bins (Fig. 15). We have applied a Kolmogorov-Smirnov test to quantify the observed differences of these functions. When the central, intermediate, and outer radial bin are compared pairwise, we obtain in each case a confidence level of more than $99 \%$ that the mass distributions do not originate from the same distribution.

Thus, the inner regions of the cluster are indeed skewed towards higher masses either by sinking of the high mass stars towards the cluster center due to dynamical processes or by primordial mass segregation, or both. The same effect is observed in the similarly young cluster NGC 3603 (Grebel et al. 2002, in prep.).

\subsection{Formation locus of massive stars}

We find several (5) high mass stars in the cluster vicinity. These stars fall onto the Arches main sequence after applying the reddening correction (Sect. 3.1). When the entire HST field is separated into two equalsize areas, the first area being a circle of radius $16^{\prime \prime}$ around the cluster center, and the second the surrounding field, no additional comparably high-mass stars are found with Arches main sequence colours except for the two bright stars found at the edges of the Gemini field. In the dynamical models of Bonnell \& Davies (1998) there is a low, but non-zero probability that a massive star originating outside the cluster's half-mass radius might remain in the cluster vicinity. High-mass stars formed near the center show, however, a tendency to migrate closer to the cluster center. The disruptive GC potential might enhance the ejection process of low-mass stars, but according to equipartition, it is unlikely that the most massive stars gain energy due to dynamical interaction with lower mass objects. We have to bear in mind, however, that interactions between massive stars in the dense cluster core could cause the ejection of high-mass stars.

$N$-body simulations performed by Portegies Zwart et al. (1999) show that the inclusion of dynamical mass segregation in cluster evolution models enhances the collision rate by about a factor of 10 as compared to theoretical cross section considerations. For a cluster with 12000 stars initially distributed according to a Scalo (1986) mass function, a relaxation time of $10 \mathrm{Myr}$, and a central density and half-mass radius comparable to the values found in Arches, about 15 merging collisions occur within the first $10 \mathrm{Myr}\left(1 t_{\text {relax }}\right)$ of the simulated cluster. Shortly after the start of the simulations, frequent binary and multiple systems form from dynamical interactions leading to the ejection of several contributing massive stars. The flat MF in the Arches center as compared to a Scalo MF, containing a larger fraction of massive stars to interact, may even increase the collision rate.

Though it is likely that the high-mass stars seen in the immediate vicinity of the Arches cluster have formed from the same molecular cloud at the same time as the cluster, a final conclusion on the possible ejection of these stars from the cluster core due to dynamical processes can only be drawn
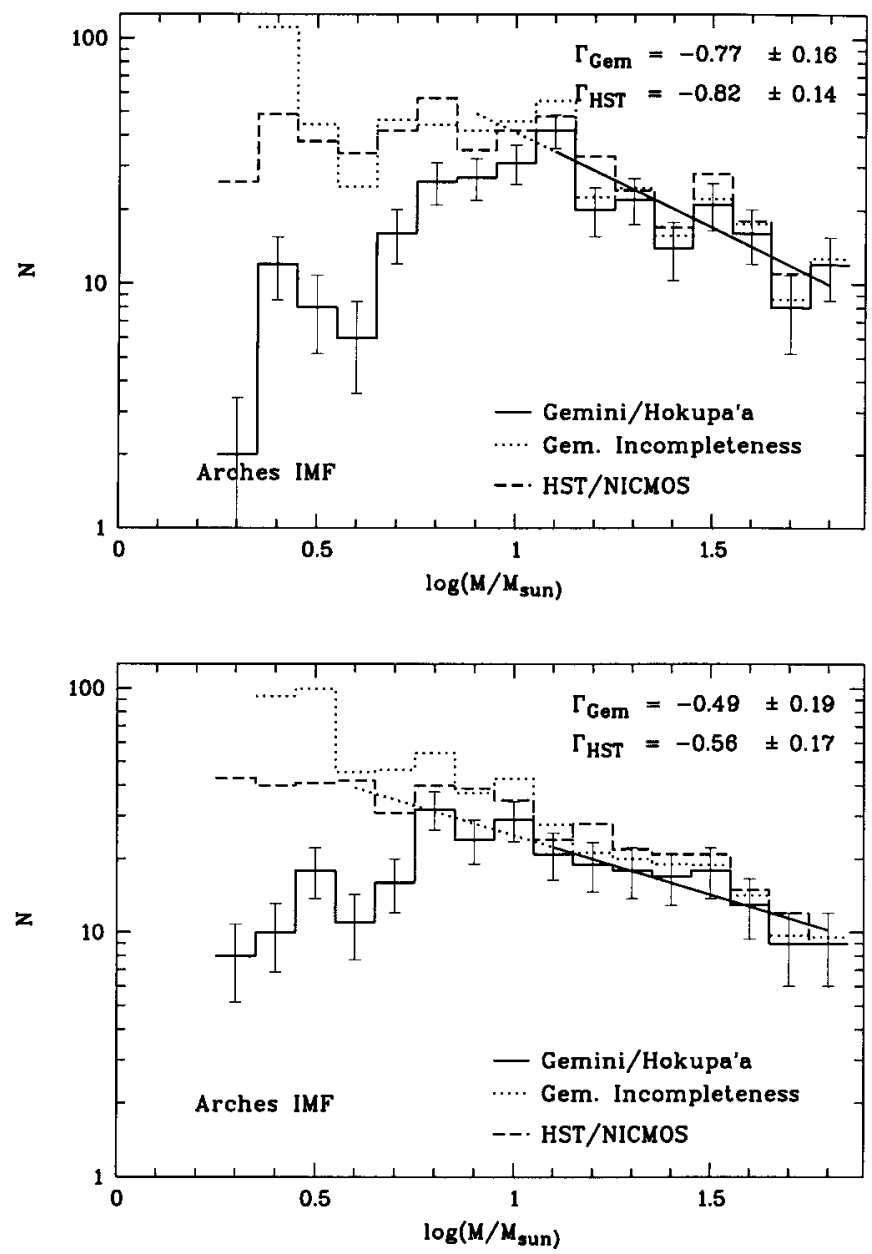

Fig. 13. Arches mass function derived from the Gemini/Hokupa'a colour-magnitude diagram shown in Fig. 9. A 2 Myr main sequence isochrone from the Geneva set of models (Lejeune \& Schaerer 2001) was used to transform magnitudes into stellar masses. The mass function has been derived for bins of $\delta \log \left(M / M_{\odot}\right)=0.1$ with a lowest mass bin $\log \left(M / M_{\odot}\right)=0.20$. Upper panel: with $A_{K}$ correction. Lower panel: without $A_{K}$ correction.

when velocities for these cluster member candidates are available.

\section{Comparison with cluster formation models}

In this section, we will first use the simple analytical approach to analyse internal cluster dynamics as summarised in Binney \& Tremaine (1987), and will then compare the derived dynamical timescales to $N$-body simulations where the tidal force of the Galactic Center potential is considered.

From the transformation of stellar magnitudes into masses, a rough estimate of the timescales relevant for dynamical cluster evolution can be made. For larger area coverage, we have used the HST/NICMOS data in the calculation below. The timescales characterising cluster evolution are the cluster's crossing time, $t_{\text {cross }}$, which is simply given by some characteristic radius divided by the average velocity, i.e., the mean velocity dispersion of the cluster, $r_{\mathrm{c}} /\langle\sigma\rangle$, and the relaxation time, $t_{\mathrm{rh}}$. The median relaxation time is the time after which gravitational encounters of stars have caused the system to equilibrate 

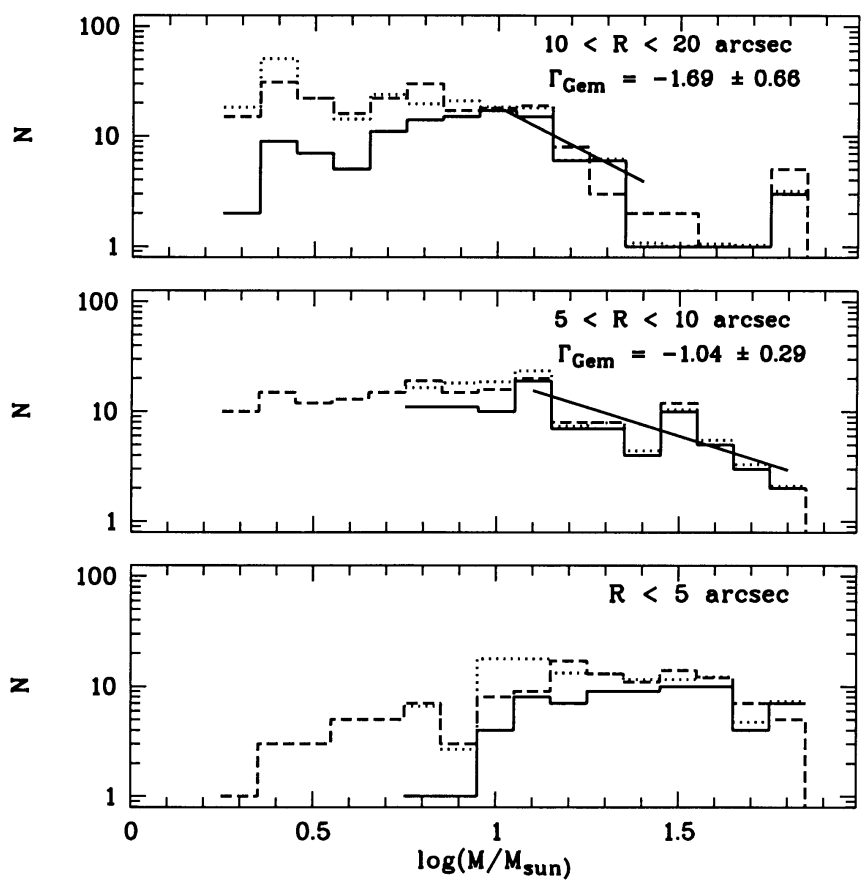

Fig. 14. Radial change of the mass function as observed in the Gemini/Hokupa'a data. A very flat mass function is seen in the inner cluster regions, where predominantly massive stars are found. The slope of the mass function increases towards the Salpeter value $(\Gamma=-1.35)$ already at a radial distance from the cluster center of $>10^{\prime \prime}(0.4 \mathrm{pc}$ at a distance of $8 \mathrm{kpc})$.

to a state independent of the original stellar orbits (Binney \& Tremaine 1987, hereafter BT87)

$t_{\mathrm{rh}}=\frac{6.63 \times 10^{8} \mathrm{yr}}{\ln (0.4 N)}\left(\frac{M}{10^{5} M_{\odot}}\right)^{1 / 2}\left(\frac{1 M_{\odot}}{m_{*}}\right)\left(\frac{r_{\mathrm{c}}}{1 \mathrm{pc}}\right)^{3 / 2}$,

where $M$ is the total mass within some characteristic radius $r_{\mathrm{c}}$, $m_{*}$ is a characteristic stellar mass, here defined as the median of the observed mass distribution, and $N$ is the number of stars in the cluster. $t_{\mathrm{rh}}$ may also be expressed in terms of the crossing time, $t_{\mathrm{rh}} \sim 0.1 \mathrm{~N} / \ln N \cdot t_{\text {cross }}$.

To evaluate the above formula, we need to know the total mass of the system, $M$, a characteristic radius, $r_{\mathrm{c}}$, the number of stars, $N$, and the characteristic stellar mass, $m_{*}$. In principle, we are able to derive most of these quantities from isochrone fitting, by assigning each star a mass corresponding to its $K$ band luminosity, and analysing the resulting spatial distribution of masses. Naturally, these individual masses cannot be accurate for each star, as they depend strongly on the choice of the isochrone and inherit the uncertainties of the photometry. The integrated properties are, however, not very sensitive to the age of the model isochrone used, within the reasonable age range of Arches, $\sim 2-3 \mathrm{Myr}$ (results will be given below). We have attempted to create a density profile from the HST/NICMOS data, but as the profile appears to be very distorted, we have chosen to use the half-mass radius, $r_{\mathrm{hm}}$, as a characteristic scale. $r_{\mathrm{hm}}$ has been obtained from the observed stellar population of the cluster under the assumption that the mass in the cluster center, relevant for the spatial scale on which gravitational interactions are important, is dominated by the

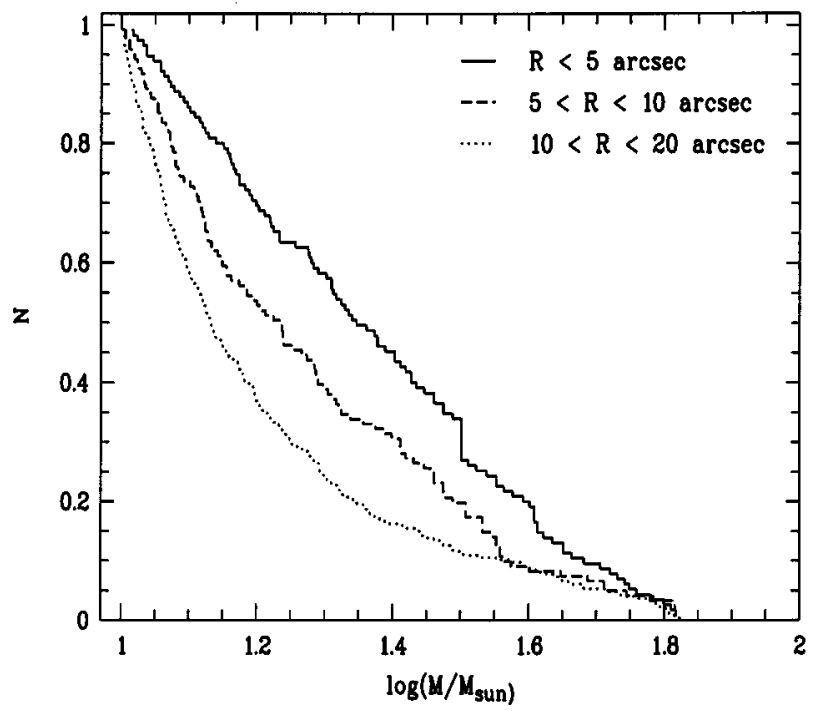

Fig. 15. Cumulative functions of the stars in each radial bin obtained from HST/NICMOS data. Each radial bin corresponds to one radial mass function in Fig. 14. The cumulative distributions have been normalised at $10 M_{\odot}$, the limit where all radii are more than $75 \%$ complete.

detected high-mass stellar population in the cluster center. The relaxation time derived from $r_{\mathrm{hm}}$ is usually referred to as the "half-mass relaxation time" (cf. Portegies Zwart et al. 2002).

The mass distribution within the cluster as derived from the isochrone allows us to estimate $r_{\mathrm{hm}}, M$, and $m_{*}$. Taking all stars within a certain radius with Arches main sequence colours as cluster members, we can also estimate $N$.

We obtain a half-mass radius of $r_{\mathrm{hm}}=10^{\prime \prime} \hat{=} 0.4 \mathrm{pc}$, irrespective of the age of the isochrone used (2 Myr or 3.2 Myr) to transform $K$-band magnitudes into masses. The total mass measured within this radius is $M\left(r_{\mathrm{hm}}\right)=6300 M_{\odot}\left(5400 M_{\odot}\right.$ for the 3.2 Myr isochrone). By extrapolation of the mass function down to $0.1 M_{\odot}$, FKM derived a total mass of $12000 M_{\odot}$ within $9^{\prime \prime}$ radius. To estimate the total mass in the cluster center, they used the relative fraction of stars with $M>40 M_{\odot}$ in two annuli separated at $R=3^{\prime \prime}$ as a scaling factor. This procedure ignores the segregation of high-mass stars. As the cluster shows strong evidence for mass segregation (see Sect. 5.3), this may overestimate the total cluster mass (within $R<9^{\prime \prime}$ ). On the other hand, our mass estimate, ignoring incompleteness and the lower mass tail, is a lower limit to the total mass within $R<10^{\prime \prime}$. We thus conclude that for an order-of-magnitude estimation, a total mass of $10^{4} M_{\odot}$ within the half-mass radius is a good approximation. This yields an average mass density of $\rho\left(R<r_{\mathrm{hm}}\right) \sim 4 \times 10^{4} M_{\odot} \mathrm{pc}^{-3}$.

The calculation of the relaxation time requires an estimate of the characteristic stellar mass and the number of stars. The median mass within $R<10^{\prime \prime}$ is $7.3 M_{\odot}$ in our observed mass range of $1.4<M<65 M_{\odot}$ in the HST sample, and the number of stars actually observed is 486 . We choose to use the median mass here as a more realistic mass estimate than the mean for individual stars participating in interaction processes, as we are aware of the fact that the mean is highly biased by the high 
fraction of high-mass stars in the cluster center. Nevertheless, we have to keep in mind that this median value is still unrealistically high as we miss the low-mass tail of the mass distribution, which also has the highest number of stars.

As the low-mass tail of the distribution is highly incomplete, we estimate $N$ to be at least $10000 M_{\odot} / 7.3 M_{\odot} \sim 1400$. This results in $t_{\mathrm{rh}}=1.2 \mathrm{Myr}$. Decreasing the characteristic mass and increasing the number of stars lengthens the relaxation time. For instance, lowering $m_{*}$ to $1 M_{\odot}$, where we take into account the fact that the cluster center is mass segregated as opposed to a standard Salpeter mass function and thus the characteristic mass of a star should be higher, and raising $N$ to $10000 \mathrm{stars}$, would result in $t_{\mathrm{rh}}=6.2 \mathrm{Myr}$. If a significant fraction of low-mass stars would have been formed and survived in the cluster center, the half-mass relaxation time would increase even more. The present-day relaxation time is thus at least on the order of or longer than the age of the Arches cluster.

A relaxation time longer than the lifetime of the cluster would imply that not yet sufficient time has passed for dynamical mass segregation to take place and would thus indicate that primordial mass segregation was present at the time of cluster formation. However, we have so far ignored the Galactic Center tidal forces accelerating the dynamical evolution of the cluster. Kim et al. (2000) performed $N$-body simulations using the observed parameters of the Arches cluster to trace the dynamical evolution of the cluster and constrain initial conditions. They use a total mass of $2 \times 10^{4} M_{\odot}$, tidal radius of $\sim 1 \mathrm{pc}$ and a single power-law IMF with slopes of $\Gamma=-0.5,-0.75,-1.0$ and -1.35 . The number of stars ranges between 2600 for a lower mass cutoff of $m_{\text {low }}=0.1 M_{\odot}$ and 12700 for $m_{\text {low }}=1 M_{\odot}$. From comparison with the HST/NICMOS data of FKM their models yield a power law with $\Gamma=-0.75$ as the most probable initial mass distribution. Although this slope is very close to the observed present-day MF, they also note that mass segregation takes place on timescales as short as $1 \mathrm{Myr}$, such that the cluster looses all memory of the initial conditions shortly after formation. Portegies Zwart et al. (2002) use a Scalo (1986) MF to model the Arches cluster, and derive a total cluster mass of $4 \times 10^{4} M_{\odot}$, and an initial relaxation time of 20-40 Myr. When comparing with the same set of HST/NICMOS observations, they conclude that a standard IMF can evolve into the current MF due to dynamical segregation, and that the IMF did not need to be overpopulated in high-mass stars. Their computations indicate that the relaxation time is strongly variable. During the dynamical evolution, $t_{\mathrm{rh}}$ increases strongly after core collapse, which occurs within $\sim 2 \mathrm{Myr}$, due to cluster reexpansion, and only starts to decrease after 10 Myr or later. The observed relaxation time does thus probably not trace the cluster's initial conditions, but reflects the current dynamical state in the evolution of Arches. In particular, a present-day relaxation time larger than the cluster's age does not necessarily imply that the cluster is not dynamically relaxed.

Unfortunately, this means that we are not able to distinguish between primordial and dynamical mass segregation from the estimated relaxation time. For the Orion Nuclear Cluster (ONC) and its core, the Trapezium, which has been studied in greater detail (e.g., Hillenbrand 1997; Hillenbrand \& Hartmann 1998), Bonnell \& Davies (1998) derive dynamical timescales too long to explain the segregation observed in high-mass stars within the Trapezium by dynamical evolution, concluding that a significant amount of primordial segregation must have been present. In the case of the Arches cluster, the external gravitational field acts towards a fast disruption, thereby impeding the equilibration process, such that dynamical segregation may well be under way.

As the different model calculations do not agree with respect to the IMF required to create the observed present-day mass distribution, a final conclusion on whether or not the Arches initial mass function had to be enriched in massive stars, thus supporting high-mass star formation models, cannot be drawn.

The evaporation time, setting the scale for dynamical dissolution by internal processes, can be estimated as $t_{\text {evap }} \approx$ $136 t_{\text {relax }} \sim 177 \operatorname{Myr}\left(857 \mathrm{Myr}\right.$ for $m_{*}=1 M_{\odot}$, BT87). Kim et al. (1999) have shown that the time required to disrupt an Arches-like cluster within the GC potential is only $10 \mathrm{Myr}$, much shorter than evaporation by internal processes will ever be relevant. The models by Portegies Zwart et al. (2002) suggest somewhat longer evaporation times in the range $30<$ $t_{\text {evap }}<50$ Myr for an Arches-like cluster. The external potential is thus the dominating factor in the dynamical evolution of Arches. Note that, however, even a cluster as dense as Arches would not survive for more than 1 Gyr independent of its locus of formation. The relaxation and evaporation times found for Arches are very similar to our results for the young, compact cluster in NGC 3603 (Grebel et al. 2002, in prep.). However, as NGC 3603 is not torn apart by additional external tidal forces, it may survive much longer than Arches. For comparison, massive young clusters in the Magellanic Clouds have relaxation times of $\sim 10^{8} \mathrm{yr}$, and corresponding evaporation times of $\sim 10^{10} \mathrm{yr}$ (e.g., Subramaniam et al. 1993), and may thus survive for one Hubble time.

\section{Conclusions}

We have analysed high-resolution Gemini/Hokupa'a adaptive optics and HST/NICMOS data of the Arches cluster near the Galactic Center with respect to spatial variations in the mass function and their implications for cluster formation. A detailed comparison of the Gemini data to HST/NICMOS observations allows us to investigate the instrumental characteristics of PSF fitting photometry with the Hokupa'a AO system.

\subsection{Technical comparison of Gemini/Hokupa'a with HST/NICMOS}

The calibration of the Gemini/Hokupa'a data of the Arches cluster using HST/NICMOS data from Figer et al. (1999) allows us to carry out a detailed technical comparison of the two datasets. Maps of photometric residuals show a strong dependence of the calibration error on the stellar density within the field. In particular, the vicinity of fainter objects to bright stars causes the Gemini magnitude to be underestimated in comparison with HST/NICMOS. This is understandable as the uncompensated seeing halos of bright stars enhance the background in a non-homogeneous way, thereby causing an overestimation of 
the background and a subsequent underestimation of the faint objects' magnitude. Conversely, the flux of very bright sources seems to be overestimated. The correlation of the photometric residual with the position of bright stars is more pronounced in $K^{\prime}$, where crowding is the dominant source of photometric uncertainty, while the effect of angular anisoplanatism is less severe. In the $H$-band the anisoplanatism is more pronounced, and hence photometric uncertainties are a blend of uncertainties due to the distance to the guide star and due to the proximity to bright sources.

The incompleteness of the luminosity function measured with Hokupa'a increases faster at fainter magnitudes than the incompleteness observed in the NICMOS LF despite the comparable detection limit in both datasets. As expected, this effect is particularly pronounced in the dense cluster center, where crowding is most severe.

As the Strehl ratio determines the amount of light scattered into the seeing induced halo around each star, a good SR is crucial to achieve not only a diffraction limited spatial resolution, but to benefit from the adaptive optics correction in dense fields containing a wide range of magnitudes. In the Arches dataset, the $S R$ of only $2.5 \%$ in $H$ and $7 \%$ in $K^{\prime}$ as compared to $95 \%$ in $F 160 \mathrm{~W}$ and $90 \%$ in $F 205 \mathrm{~W}$ (NICMOS) is clearly the limiting factor for crowded field photometry. As Hokupa'a was initially designed and developed for the $3.6 \mathrm{~m} \mathrm{CFH}$ telescope, its performance at the $8 \mathrm{~m}$ Gemini telescope is naturally constrained by the limited number of only 36 actuators. In the case of the Arches science demonstration data, additional constraints were given by the seeing, the high airmass due to the low latitude of the Galactic Center, and the guide star magnitude. Under better observing conditions and with a brighter guide star Strehl ratios of up to $30 \%$ can be achieved with Hokupa'a at Gemini. Higher order AO systems are currently capable to produce SRs of up to $50 \%$.

The Gemini ground-based AO data are comparable to the HST/NICMOS data in the resolution of bright sources $\left(K^{\prime}<\right.$ $18 \mathrm{mag}$ ), and in a non-crowded field. They do reach their limitations in the densest cluster area and in the case where faint stars are located close to a bright object. Higher Strehl ratios would of course reduce this unequality.

\subsection{Photometric results}

A strong colour gradient is detected over the field of the Arches cluster, revealing an increase in visual extinction of approximately $9<\Delta A_{V}<15$ mag when progressing outwards from the cluster center. The visual extinction is estimated from the Rieke \& Lebofsky (1985) extinction law to be $A_{V} \sim 24$ mag in the cluster center, increasing to a maximum of $33<A_{V}<$ 39 mag in the vicinity, in accordance with Cotera et al. (2000), who found a maximum of $A_{V}=37 \mathrm{mag}$ in the Arches field. Within the central $5^{\prime \prime}$ radius, however, no colour gradient is observed. This indicates that the cluster center has been stripped of the remaining dust and gas either by strong stellar winds from massive stars or by photo-evaporation, or both. Beyond 5", a linear increase in extinction is observed, suggesting an increasing amount of dust with distance from the cluster center. Photo-evaporation due to the strong UV radiation of the $8 \mathrm{WN} 7 / 8$-stars and more than $100 \mathrm{O}$-stars found in the cluster center is most probably responsible for dust dissolution.

The $m 160-m 205, m 205$ (equivalent to $H-K, K$ ) colourmagnitude diagrams derived from the HST and Gemini data sets both show a bent main sequence following this colour trend. The main sequence straightens out when correcting for this colour variation. A spatial analysis of the CMDs reveals the bulk of the bright stars on the Arches field to be located in the cluster center.

\subsection{Mass functions}

Present-day mass functions have been derived from the CMDs after linear correction of the colour trend and corresponding change in extinction over the field, and selection of a reasonable main sequence colour cut. The integrated mass function derived from the Gemini photometry displays a slope of $\Gamma=-0.8 \pm 0.15$ for $6<M<65 M_{\odot}$, less steep than the Salpeter slope of $\Gamma=-1.35$. This value agrees with the slopes derived from the HST data in the same manner (Sect. 5), and with the values presented in FKM within the uncertainties. When the magnitudes are not corrected for differential extinction, the slope of the MF is significantly flatter, $\Gamma \sim-0.5 \pm 0.2$. Particularly in young star forming regions, the effects of differential extinction are thus clearly non-negligible.

The analysis of the radial dependence of the mass function reveals a very flat IMF in the immediate cluster center with a slope close to zero. The IMF slope seems to increase outwards with $\Gamma=-1.0 \pm 0.3$ for $5<R<10^{\prime \prime}$ and $\Gamma=-1.7 \pm 0.7$ beyond $R>10^{\prime \prime}$. We have created cumulative functions for the stars in each radial bin, and performed a KS test to derive the significance level of the variance in the mass distributions. The probability for the observed distributions to originate in the same mass function is below $1 \%$ when comparing each two of the three radial bins analysed.

\subsection{Cluster dynamics}

The flat mass function in the Arches center is a strong indication for mass segregation. While the center seems to be dominated by high-mass stars, the cluster edges display the standard behaviour of a young stellar population. A similar radial dependence of the mass function is observed in the young, compact cluster NGC 3603 (Grebel et al. 2002, in prep.), located in a normal star forming environment in the Carina spiral arm. The fact that two out of three compact young clusters found in the Milky Way, which have been analysed in such detail to date, display a flat mass function slope in the core indicates that such a behaviour might be typical for starburst clusters and is not restricted to the extreme GC environment.

Mass segregation in a compact, massive cluster can either be caused by an enhanced production efficiency of massive stars, or by dynamical segregation during the cluster's evolution. We roughly estimate the present-day relaxation time of Arches from mass function considerations to be about a few Myr ( $\left.\sim 20 t_{\text {cross }}\right)$, and thus of the same order of magnitude as 
the cluster age of 2 Myr. Again, a similar timescale has been found for NGC 3603 as well (Grebel et al. 2002, in prep.). The comparison with $N$-body simulations suggests, however, that the dynamical evolution of massive clusters close to the Galactic Center whipes out the initial conditions within less than 1 Myr. We are therefore not able to distinguish between primordial and dynamical mass segregation. Both effects are intertwined at the current state of cluster evolution, such that a detailed dynamical analysis is crucial for a thorough understanding of the formation process. This analysis has to await deep, high resolution infrared spectroscopy to obtain radial velocities and proper motions for a significant fraction of stars belonging to the cluster population.

Acknowledgements. We are grateful to the Gemini Team for providing the science demonstration data, as well as for all kinds of useful information and support on data analysis issues. In particular, we would like to thank Dr. François Rigaut, Dr. Jean-René Roy and Dr. Mark Chun for their help with data reduction problems. We thank Dr. Michael Odenkirchen for infinite patience in helpful discussions. We also thank our referee Simon Portegies Zwart for discussions and useful comments which helped to improve the paper, in particular with respect to the mass function discussion and dynamical considerations.

The work presented here is based on observations obtained at the Gemini Observatory, which is operated by the Association of Universities for Research in Astronomy, Inc., under a cooperative agreement with the NSF on behalf of the Gemini partnership: the National Science Foundation (United States), the Particle Physics and Astronomy Research Council (UK), the National Research Council (Canada), CONICYT (Chile), the Australian Research Council (Australia), CNPq (Brazil) and CONICET (Argentina), and based on observations made with the NASA/ESA Hubble Space Telescope, obtained at the Space Telescope Science Institute, which is operated by the Association of Universities for Research in Astronomy, Inc., under NASA contract NAS 5-26555. These observations are associated with proposal No. 7364.

\section{References}

Behrend, R., \& Maeder, A. 2001, A\&A, 373, 190

Binney, J., \& Tremaine, S. 1987, Galactic Dynamics (Princeton University Press), 514

Blum, R. D., Schaerer, D., Pasquali, A., et al. 2001, AJ, 122, 1875

Bonnell, I. A., \& Davies, M. B. 1998, MNRAS, 295, 691

Bonnell, I. A., Bate, M. R., \& Zinnecker, H. 1998, MNRAS, 298, 93

Brandner, W., Grebel, E. K., Barbá, R. H., et al. 2001, AJ, 122, 858 (BGB)

Carr, J. S., Sellgren, K., \& Balachandran, S. C. 2000, ApJ, 530, 307

Cotera, A. S., Erickson, E. F., Colgan, S. W. J., et al. 1996, ApJ, 461, 750

Cotera, A. S., Simpson, J. P., Erickson, E. F., et al. 2000, ApJS, 129, 123

Crowther, P. A., Smith, L. J., Hillier, D. J., \& Schmutz, W. 1995, A\&A, 293, 427

Devillard, N. 1997, The messenger No. 87

Dutra, C. M., \& Bica, E. 2000, A\&A, 359, 347

Dutra, C. M., \& Bica, E. 2001, A\&A, 376, 434
Eisenhauer, F., Quirrenbach, A., Zinnecker, H., et al. 1998, ApJ, 498, 278

Elmegreen, B. 1999, ApJ, 515, 323

Elmegreen, B. 2001, in The Central kpc of Starbursts and AGN, ed. J. H. Knapen, J. E. Beckman, I. Shlosman, \& T. J. Mahoney, ASP Conf. Ser., 249, 492

Figer, D. F., Kim, S. S., Morris, M., et al. 1999, ApJ, 525, 750 (FKM)

Frogel, J. A., \& Whitford, A. E. 1987, ApJ, 320, 199

Frogel, J. A., Tiede, G. P., \& Kuchinski, L. E. 1999, AJ, 117, 2296

Graves, J. E., Northcott, M. J., Roddier, F. J., et al. 2000, SPIE, 4007, 126

Grebel, E.K., Brandner, W., \& Roberts, W. J. 1996, A\&A, 311, 470

Grebel, E. K., \& Chu, Y.-H. 2000, AJ, 119, 787

Hillenbrand, L. A. 1997, AJ, 113, 1733

Hillenbrand, L. A., \& Hartmann, L. W. 1998, ApJ, 492, 540

Hodapp, K.-W., Hora, J. L., Hall, D. N. B., et al. 1996, New Astron., 1,177

Howarth, I. D., \& Prinja, R. K. 1989, ApJS, 96, 527

Kim, S. S., Morris, M., \& Lee, H. M. 1999, ApJ, 525, 228

Kim, S. S., Figer, D. F., Lee, H. M., \& Morris, M. 2000, ApJ, 545, 301

Kroupa, P. 2001, MNRAS, 322, 231

Lang, C. C., Goss, W. M., \& Morris, M. 2001, AJ, 121, 2681

Larson, R. B. 1982, MNRAS, 200, 159

Lejeune, T., \& Schaerer, D. 2001, A\&A, 366, 538

Massey, P., Lang, C. C., DeGioia-Eastwood, K., \& Garmany, C. D. 1995b, ApJ, 438, 188

Massey, P., Johnson, K. E., \& DeGioia-Eastwood, K. 1995a, ApJ, 454, 151

McNamara, D. H., Madsen, J. B., Barnes, J., \& Ericksen, B. F. 2000, PASP, V112, Issue 768, 202

Morris, M. 1993, ApJ, 408, 496

Palla, F., \& Stahler, F. W. 1999, ApJ, 525, 772

Portegies Zwart, S. F., Makino, J., McMillan, S. L. W., \& Hut, P. 1999, A\&A, 348, 117

Portegies Zwart, S. F., Makino, J., McMillan, S. L. W., \& Hut, P. 2001, ApJ, 546, L101

Portegies Zwart, S. F., Makino, J., McMillan, S. L. W., \& Hut, P. 2002, ApJ, 565, 265

Ramirez, S. V., Sellgren, K., Carr, J. S., et al. 2000, ApJ, 537, 205

Rich, R. 1988, AJ, 95, 828

Rieke, G. H., \& Lebofsky, M. J. 1985, ApJ, 288, 618

Salpeter, E. E. 1955, ApJ, 121, 161

Scalo, J. M. 1986, Fund. Cosmic Phys., 11, 1

Scalo, J. M. 1998, in The stellar initial mass function, ASP Conf. Ser., 142,201

Schaerer, D., Meynet, G., Maeder, A., \& Schaller, G. 1993, A\&AS, 98, 523

Schaller, G., Schaerer, D., Meynet, G., \& Maeder, A. 1992, A\&AS, 96, 269

Smith, L. J., \& Maeder, A. 1989, A\&A, 211, 71

Stetson, P. B. 1987, PASP, 99, 191

Stetson, P. B. 1990, PASP, 102, 932

Subramaniam, A., Sagar, R., \& Bhatt, H. C. 1993, A\&A, 273, 100

Terndrup, D. M., Frogel, J. A., \& Whitford, A. E. 1990, ApJ, 357, 453

Terndrup, D. M., Frogel, J. A., \& Whitford, A. E. 1991, ApJ, 378, 742

Tody, D. 1993, in Astronomical Data Analysis Software and Systems II, ed. R. J. Hanisch, R. J. V. Brissenden, \& J. Barnes, ASP Conf. Ser., 52, 173

Yorke, H. W., \& Krügel, E. 1977, A\&A, 54, 183 